\title{
INGESTION DES HÉMATOZOAIRES PAR LE VECTEUR.
}

\author{
Analyse de quatre Filaires parasites d'un saïmiri \\ Deuxième partie * \\ G. PETIT**
}

\section{Chapitre VI : Ingestion des microfilaires}

\section{A - Ingestion pendant le creux diurne de la microfilarémie}

Le déroulement de l'expérience est le suivant :

- anesthésie du Saïmiri à $9 \mathrm{~h}$ (première anesthésie),

- mesure de la microfilarémie avant le repas des Aedes, au flanc gauche (9 h 07) et à l'oreille gauche $(9 \mathrm{~h} 10)$,

- gorgement des Aedes sur le flanc droit entre 9 h 10 et 9 h 20,

- mesure de la microfilarémie après le repas des Aedes, à l'oreille droite ( 9 h 20),

- estimation par pesée du volume moyen de sang ingéré (cf. chap. Méthodes) : $2,85 \mathrm{~mm}^{3} /$ Aedes.

Bien que l'expérience ait été aussi brève que possible, l'effet de l'anesthésique sur les microfilarémies a déjà commencé; les densités microfilariennes mesurées à l'oreille avant et après le repas sont les suivantes :
D. robini,
$47-80$
D. gracile,
$121-135$
M. (T.) mariae,
$91-85$
$M$. (T.) colombiensis,
$17-21$

Il faut donc tenir compte de cette évolution des microfilarémies pour établir la relation entre le nombre de microfilaires ingérées et la densité microfilarienne dans le sang prélevé par l'expérimentateur.

a) Estimation de la densité microfilarienne moyenne au moment du repas des Aedes.

Le singe est placé dix minutes sur la cage, mais les moustiques ne se gorgent pas immédiatement; nous considérons que l'heure moyenne du gorgement se situe

\footnotetext{
- La première partie de cet article a été publiée dans les Annales de Parasitologie, 1985, 60, nª 3, 247-297.

-* Laboratoire de Zoologie - Vers, Muséum National d'Histoire Naturelle, 61 rue Buffon, F 75231 Paris Cedex 05.

Accepté le 14 juin 1984 .
} 
$7^{\prime}$ après la pose du Saimiri. A ce moment, la densité microfilarienne probable à l'oreille est donc : densité avant le repas $+2 / 3$ de la différence entre les densités avant et après le repas.

Pour obtenir la valeur de la microfilarémie au flanc au même moment, le calcul est différent puisque nous ne disposons que d'une seule mesure effectuée avant le repas; nous admettons comme approximation une valeur calculée de la façon suivante : microfilarémie au flanc avant le repas augmentée du pourcentage de la variation telle qu'elle a été estimée à l'oreille.

b) Calcul du rapport entre la densité microfilarienne dans le sang prélevé par l'Aedes et dans celui prélevé par l'expérimentateur (tableau $X X I X)$.

Les densités microfilariennes sont rapportées à $10 \mathrm{~mm}^{3}$ aussi bien pour le sang prélevé par l'expérimentateur que pour le sang ingéré par l'Aedes.

Deux types de rapports sont calculés, l'un en fonction de la densité microfilarienne au flanc et l'autre de la densité microfilarienne à l'oreille.

TABleaU XXIX. - Ingestion au flanc des microfilaires de $S$. sciureus par A. aegypti pendant le creux diurne de microfilarémie (15 Aedes). Anesthésique à $9 \mathrm{~h}-10$ octobre 1983 .

\begin{tabular}{|c|c|c|c|c|}
\hline & D. robini & D. gracile & $\begin{array}{c}M .(T .) \\
\text { mariae }\end{array}$ & $\begin{array}{c}M .(T .) \\
\text { colombiensis }\end{array}$ \\
\hline $\begin{array}{l}\text { Mf I F / A } \\
\text { Mf I F / } 10 \mathrm{~mm}^{3} \\
\text { Mf F / } 10 \mathrm{~mm}^{3} \\
\text { Mf I } / \mathrm{Mf} \mathrm{F}\end{array}$ & $\begin{array}{l}27+/-11 \\
95+1-11 \\
101 \\
\mathrm{R} 21=0,94\end{array}$ & $\begin{array}{c}54+/-10 \\
188+1-14 \\
170 \\
\mathrm{R} 22=1,11\end{array}$ & $\begin{array}{c}33+1-10 \\
117+1-10 \\
123 \\
\mathrm{R} 23=0,95\end{array}$ & $\begin{array}{c}0,26+/-0,34 \\
0,9+/-1,2 \\
0,57 \\
\mathrm{R} 24 \stackrel{1,58}{=}\end{array}$ \\
\hline $\begin{array}{l}\text { Mf O/ } 10 \mathrm{~mm}^{3} \\
\text { Mf I F / Mf O }\end{array}$ & $\mathrm{R} 25 \stackrel{69}{=} 1,38$ & $\mathrm{R} 26 \stackrel{130}{=} 1,45$ & $\mathrm{R} 27 \stackrel{87}{=} 1,35$ & $\mathrm{R} 28 \stackrel{20}{=} 0,05$ \\
\hline
\end{tabular}

Mf $\mathrm{I}=$ nombre moyen de microfilaires ingérées

Mf $=$ microfilarémie

$\mathrm{O}=$ oreille, $\mathrm{F}=$ flanc, $\mathrm{A}=$ Aedes

- Pour D. robini, D. gracile, et $M$. (T.) mariae, la densité des microfilaires dans le sang ingéré par l'Aedes au flanc est peu différente de la densité des microfilaires dans le sang prélevé par l'expérimentateur au flanc (R21 à R23 = 1). Par contre elle est nettement supérieure à la densité des microfilaires dans le sang prélevé par l'expérimentateur à l'oreille (R25 à R27 = 1,30).

- Pour $M$. (T.) colombiensis, le rapport d'ingestion R24 a peu de signification étant donné la faiblesse de la microfilarémie au flanc. Le rapport R28 exprime la rareté des microfilaires de cette espèce au flanc (voir R4 chap. v).

L'interprétation de ces résultats est donnée dans le chapitre viI. 


\section{B - Ingestion pendant le pic de la microfilarémie}

L'expérience s'est déroulée de la façon suivante :

- anesthésie du Saimiri à $9 \mathrm{~h}$ (première anesthésie),

- mesure de la microfilarémie avant le repas des Aedes, au flanc droit (10 h 33 ) et à l'oreille $(10$ h 35$)$,

- gorgement des Aedes sur le flanc gauche,

- mesure de la microfilarémie après le repas des Aedes, au flanc droit ( $11 \mathrm{~h}$ 02) et à l'oreille $(11 \mathrm{~h} \mathrm{05})$,

_- estimation par pesée (cf. Méthodes) du volume moyen de sang ingéré : $3,72 \mathrm{~mm}^{3}$ par Aedes.

Comme dans l'expérience précédente, la microfilarémie n'est pas restée stable. Les incisions du flanc, et le gorgement des moustiques, effectués ici $1 \mathrm{~h} 30$ après l'anesthésie, ont déclenché une agitation assez forte du singe sans toutefois le réveiller. Il semble que cette perturbation du sommeil de l'animal soit responsable des différences de microfilarémies observées avant et après le repas :

\begin{tabular}{|c|c|c|c|c|c|}
\hline $0 . r$ & 14 & - & 1 & - & $\mathbf{e}$ \\
\hline D. $g$ & & & 514 & & ille \\
\hline M. (T.) mariae, & 14 & & 63 au flanc et 97 & & 73 a 10 \\
\hline
\end{tabular}

Les valeurs de la microfilarémie au flanc et à l'oreille au moment du repas des Aedes sont obtenues par la même méthode que dans l'expérience précédente.

Dans le tableau $X X X$, les mêmes observations que lors du creux de microfilarémie peuvent être faites au pic.

L'interprétation de ces résultats est donnée dans le chapitre viI.

TABLeaU XXX. - Ingestion au flanc des microfilaires de $S$. sciureus par $A$. aegypti pendant le pic de microfilarémie $(16$ Aedes). Anesthésique à $9 \mathrm{~h}-14$ octobre 1983 .

\begin{tabular}{|c|c|c|c|c|}
\hline & D. robini & D. gracile & $M .(T$.$) mariae$ & $\begin{array}{c}M .(T .) \\
\text { colombiensis }\end{array}$ \\
\hline $\begin{array}{l}\text { Mf I F / A } \\
\text { Mf I F / } 10 \mathrm{~mm}^{3} \\
\text { Mf F / } 10 \mathrm{~mm}^{3} \\
\text { Mf I F / Mf F }\end{array}$ & $\begin{array}{c}525+1-11 \\
1421+1-11 \\
1146 \\
\mathrm{R} 29=1,24\end{array}$ & $\begin{array}{c}273+/-12 \\
734+/-12 \\
687 \\
\mathrm{R} 30=1,07\end{array}$ & $\begin{array}{c}29+1-18 \\
78+1-18 \\
89 \\
\mathrm{R} 31=0,88\end{array}$ & $\begin{array}{c}0,33+/=0,34 \\
0,83+/=0,85 \\
3,55 \\
\mathrm{R} 32=0,23\end{array}$ \\
\hline $\begin{array}{l}\text { Mf O / } 10 \mathrm{~mm}^{3} \\
\text { Mf I F / Mf O }\end{array}$ & $\mathrm{R} 33 \stackrel{879}{=} 1,62$ & $\mathrm{R} 34 \stackrel{558}{=} 1,32$ & $\mathrm{R} 35 \stackrel{61}{=} 1,46$ & $\begin{array}{c}6,82 \\
\mathrm{R} 36 \stackrel{1}{=} 0,12\end{array}$ \\
\hline
\end{tabular}

Mf $\mathrm{I}=$ nombre moyen de microfilaires ingérées

Mf $=$ microfilarémie

$\mathrm{O}=$ oreille, $\mathrm{F}=$ flanc, $\mathrm{A}=$ Aedes 


\section{C - Variations de l'ingestion suivant le lieu de gorgement}

1) Comparaison flanc-oreille-queue; expérience préliminaire

Des Aedes sont mis à gorger simultanément sur le Saïmiri, respectivement sur le flanc, la face ventrale de l'oreille, et les trois derniers cm de la queue. Deux séries de gorgement sont effectuées, la première à $21 \mathrm{~h}$, après anesthésie, la deuxième à $9 \mathrm{~h}$ le lendemain matin, après une nouvelle anesthésie.

La mesure de la microfilarémie est effectuée avant chaque repas à l'oreille (21 h et $9 \mathrm{~h})$, au flanc ( $21 \mathrm{~h} 05$ et $9 \mathrm{~h} 05)$, et à l'extrémité de la queue ( $21 \mathrm{~h} 10$ et $9 \mathrm{~h} \mathrm{10}$ ). Les repas ont lieu respectivement entre $21 \mathrm{~h} 10$ et $21 \mathrm{~h} 25$ et entre $9 \mathrm{~h} 10$ et $9 \mathrm{~h} 25$ avec 15 Aedes gorgés à réplétion dans chaque lot.

Les nombres moyens de microfilaires ingérées sont exprimés pour $10 \mathrm{~mm}^{3}$ en estimant que chaque Aedes a ingéré $3,5 \mathrm{~mm}^{3}$ de sang (pas de pesée).

Dans les tableaux $X X X I$ à $X X X I V$, nous comparons les rapports flanc/oreille et queue/oreille, des microfilarémies d'une part, et des nombres moyens de microfilaires ingérées d'autre part. Nous nous intéressons surtout à ces derniers rapports qui ont une grande valeur du fait que les gorgements sont simultanés.

TABleau XXXI a et b. - D. robini. Ingestion des microfilaires par $A$. aegypti; comparaison flanc - oreille - queue (15 Aedes par organe).

D. robini,

a - 20 juin 1983 anesthésique à $21 \mathrm{H}$

\begin{tabular}{|c|c|c|c|}
\hline & Flanc & Oreille & Queue \\
\hline Mf $/ 10 \mathrm{~mm}^{3}$ & $1395+/-11$ & $1019+/-11$ & $1510+1-42$ \\
\hline$\frac{\text { Mf F ou } Q}{\text { Mf O }}$ & $\mathrm{R} 37=1,37$ & & $\mathrm{R} 39=1,48$ \\
\hline Mf I $/ 10 \mathrm{~mm}^{3}$ & $1797+1-10$ & $1188+/-13$ & $769+1-12$ \\
\hline$\frac{\text { Mf I F ou Q }}{\text { Mf I O }}$ & $\mathrm{R} 38=1,51$ & & $\mathrm{R} 40=0,64$ \\
\hline \multirow[t]{2}{*}{ b - 21 juin 1983} & lésique à $9 \mathrm{H}$ & & \\
\hline & Flanc & Oreille & Queue \\
\hline $\mathrm{Mf} / 10 \mathrm{~mm}^{3}$ & $46+1-14$ & $40+1-14$ & $69+1-32$ \\
\hline Mf $F$ ou $Q$ & $\mathrm{R} 41=1,15$ & & $\mathrm{R} 43=1,72$ \\
\hline Mf O & & & \\
\hline Mf I $/ 10 \mathrm{~mm}^{3}$ & $60+1-14$ & $34+/-15$ & $29+1-26$ \\
\hline$\frac{\text { Mf I F ou } Q}{\text { Mf I O }}$ & $\mathrm{R} 42=1,75$ & & $\mathrm{R} 44=0,83$ \\
\hline
\end{tabular}

Mf $\mathbf{I}=$ nombre moyen de microfilaires ingérées

$\mathrm{Mf}=$ microfilarémie

$\mathrm{Q}=$ queue, $\mathrm{O}=$ oreille, $\mathrm{F}=$ flanc 


\section{-Comparaison flanc-oreille}

Les Aedes gorgés sur le flanc ingèrent plus de microfilaires que ceux gorgés sur l'oreille : 1,75 fois plus à $9 \mathrm{~h}(\mathrm{R} 42)$ et 1,51 fois plus à $21 \mathrm{~h}$ (R38).

\section{- Comparaison quewe-oreille}

Les Aedes gorgés sur la queue ingèrent moins de microfilaires que ceux gorgés sur l'oreille à $9 \mathrm{~h}(\mathrm{R} 44=0,83)$, à $21 \mathrm{~h}(\mathrm{R} 40=0,64)$.

TABLEAU XXXII a et b. - - D. gracile. Ingestion des microfilaires par A. aegypti; comparaison flanc-oreille-queue.

D. gracile,

a - 20 juin 1983 anesthésique à $21 \mathrm{H}$

\begin{tabular}{|c|c|c|c|}
\hline & Flanc & Oreille & Queue \\
\hline $\mathrm{Mf} / 10 \mathrm{~mm}^{3}$ & $691+1-11$ & $240+/-12$ & $669+1-45$ \\
\hline$\frac{\text { Mf F ou } Q}{\text { Mf } O}$ & $\mathrm{R} 45=1,48$ & & $\mathrm{R} 47=1,44$ \\
\hline Mf I / $10 \mathrm{~mm}^{3}$ & $826+/-11$ & $194+/-22$ & $404+/-16$ \\
\hline$\frac{\text { Mf I F ou } Q}{\text { Mf I O }}$ & $\mathrm{R} 46=1,28$ & & $\mathrm{R} 48=0,62$ \\
\hline $\mathrm{b}-21$ juin 1983 & anesthésique à $9 \mathrm{~h}$ & & \\
\hline
\end{tabular}

\begin{tabular}{lccc}
\hline & Flanc & Oreille & Queue \\
\hline Mf / 10 mm & $54+/-14$ & $76+/-13$ & $73+/-32$ \\
Mf F ou Q & $\mathrm{R} 49=0,71$ & & $\mathrm{R} 51=0,96$ \\
Mf O & $103+/-14$ & $77+/-10$ & $49+1-21$ \\
Mf I / 10 mm & & $\mathrm{R} 52=0,63$ \\
Mf I F ou Q & $\mathrm{R} 50=1,33$ & & \\
\hline Mf I O & & & \\
\hline
\end{tabular}

Mf $\mathrm{I}=$ nombre moyen de microfilaires ingérées

Mf $=$ microfilarémie

$\mathrm{Q}=$ queue $; \mathrm{O}=$ oreille $\mathrm{F}=$ flanc

\section{- Comparaison flanc-oreille}

Les Aedes gorgés sur le flanc ingèrent plus de microfilaires que ceux gorgés sur l'oreille : 1,33 fois plus à $9 \mathrm{~h}(\mathrm{R} 50)$ et 1,28 fois plus à $21 \mathrm{~h}(\mathrm{R} 46)$. C'est le même phénomène que pour $D$. robini, mais moins fort. 
- Comparaison queue-oreille

Comme avec D. robini, les Aedes gorgés sur la queue ingèrent moins de microfilaires que ceux gorgés sur l'oreille : à 9 h $\mathrm{R} 52=0,63$; à 21 h, R48 =0,62.

TableaU XXXIII a et b. - $M$. (T.) mariae. Ingestion des microfilaires par $A$. aegypti; comparaison flanc-oreille-queue.

M. (T.) mariae, a - 20 juin 1983

anesthésique à $21 \mathrm{~h}$

\begin{tabular}{|c|c|c|c|}
\hline & Flanc & Oreille & Queue \\
\hline Mf $/ 10 \mathrm{~mm}^{3}$ & $408+1-12$ & $240+1-12$ & $365+1-42$ \\
\hline$\frac{\text { Mf } F \text { ou } Q}{M f O}$ & $\mathrm{R} 53=1,7$ & & $\mathrm{R} 55=1,52$ \\
\hline Mf I $/ 10 \mathrm{~mm}^{3}$ & $408+1-13$ & $194+1-22$ & $114+1-25$ \\
\hline$\frac{\text { Mf I F ou } Q}{\text { Mf I O }}$ & $\mathrm{R} 54=2,1$ & & $\mathrm{R} 56=0,59$ \\
\hline \multirow[t]{2}{*}{ b -21 juin 1983} & sique à $9 \mathrm{~h}$ & & \\
\hline & Flanc & Oreille & Queue \\
\hline Mf $/ 10 \mathrm{~mm}^{3}$ & $64+1-13$ & $81+/-13$ & $113+/-32$ \\
\hline$\frac{\text { Mf F ou } Q}{\text { Mf } O}$ & $\mathrm{R} 57=0,79$ & & $\mathrm{R} 59=1,40$ \\
\hline Mf I / $10 \mathrm{~mm}^{3}$ & $86+/-17$ & $83+/-10$ & $43+1-21$ \\
\hline$\frac{\text { Mf I F ou } Q}{\text { Mf I O }}$ & $\mathrm{R} 58=1,03$ & & $\mathrm{R} 60=0,52$ \\
\hline
\end{tabular}

Mf I = nombre moyen de microfilaires ingérées

Mf $=$ microfilarémie

$\mathrm{Q}=$ queue $; \mathrm{O}=$ oreille $; \mathrm{F}=$ flanc

\section{- Comparaison flanc-oreille}

Comme pour $D$. robini et $D$. gracile, les Aedes ingèrent plus de microfilaires au flanc mais seulement lors du pic de microfilarémie $(\mathrm{R} 54=2,1)$. Pendant le creux ils ingèrent la même quantité de microfilaires au flanc et à l'oreille $(\mathrm{R} 58=1,03)$.

\section{- Comparaison queue-oreille}

Comme pour $D$. robini et $D$. gracile, les Aedes ingèrent moins de microfilaires à la queue : à $9 \mathrm{~h} \mathrm{R60}=0,52 ;$ à $21 \mathrm{~h}$ R56 $=0,59$. 
TABLEAU XXXIV a et b. $-M$. (T.) colombiensis. Ingestion des microfilaires par $A$. aegypti; comparaison flanc-oreille-queue.

M. (T.) colombiensis,

$\mathrm{b}-20$ juin 1983 anesthésique à $21 \mathrm{~h}$

\begin{tabular}{lccc}
\hline & Flanc & Oreille & Queue \\
\hline Mf $/ 10 \mathrm{~mm}^{3}$ & $0,22+/-122$ & $19+/-29$ & $100+/-32$ \\
Mf F ou Q & $\mathrm{R} 61=0,01$ & & $\mathrm{R} 63=5,26$ \\
Mf O & & & $1,8+1-31$ \\
Mf I / 10 mm & $1,6+/-0,9$ & $1,6+/-34$ & $\mathrm{R} 64=1,28$ \\
Mf I F ou Q & $\mathrm{R} 62=1$ & &
\end{tabular}

b - 21 juin 1983 anesthésique à $9 \mathrm{~h}$

\begin{tabular}{lccc}
\hline & Flanc & Oreille & Queue \\
\hline Mf I $10 \mathrm{~mm}^{3}$ & $1+/-56$ & $6+/-35$ & $11+/-37$ \\
Mf F ou Q & $\mathrm{R} 65=0,17$ & & $\mathrm{R} 67=1,83$ \\
Mf O & & & $2+/-25$ \\
Mf I / $10 \mathrm{~mm}^{3}$ & $1,5+/-0,8$ & $2,4+/-18$ & $\mathrm{R} 68=0,77$ \\
Mf I F ou Q & $\mathrm{R} 66=0,62$ & & \\
\hline Mf I O & & & \\
\hline
\end{tabular}

Mf $I=$ nombre moyen de microfilaires ingérées

Mf $=$ microfilarémie

$\mathrm{Q}=$ queue $; \mathrm{O}=$ oreille $; \mathrm{F}=$ flanc

Les rapports représentés ici ont très peu de signification étant donné l'importance des intervalles de confiance qui existent sur la mesure de la microfilarémie ou le nombre moyen de microfilaires ingérées.

Nous remarquons cependant que, quel que soit l'organe, les doses de microfilaires ingérées sont faibles et à peu près équivalentes.

Pour les 4 Filaires du Saïmiri, les résultats représentés dans les 4 tableaux précédents seront interprétés dans le chapitre vir.

\section{2 - Contrôle de l'expérience précédente}

L'expérience est réalisée cette fois avec pesée des moustiques; le pic de microfilarémie est provoqué par l'injection d'anesthésique. Le protocole expérimental est le suivant :

- anesthésie du singe à $9 \mathrm{~h}$ (première anesthésie),

- mesure de la microfilarémie avant le repas des Aedes, au flanc (10 h 33) et à la queue (10 h 40), 
- gorgement simultané des Aedes au flanc (16 우) et à l'extrémité de la queue (10 ㅇ),

- mesure de la microfilarémie après le repas, au flanc (11 h 02) et à la queue (11 h 08),

— volume de sang ingéré à la queue $=3,37 \mathrm{~mm}^{3}$ par Aedes; au flanc $=3,72 \mathrm{~mm}^{3}$ par Aedes.

Dans le tableau $X X X V$ nous comparons les nombres moyens de microfilaires ingérées au flanc et à la queue (rapportés à $10 \mathrm{~mm}^{3}$ ).

TableaU XXXV. - Ingestion des microfilaires de S. sciureus par A. aegypti; comparaison flanc-queue.

Anesthésie à $9 \mathrm{~h}-14$ octobre 1983

\begin{tabular}{lcccc}
\hline & D. robini & D. gracile & $M .(T$.$) mariae$ & $\begin{array}{c}M .(T .) \\
\text { colombiensis }\end{array}$ \\
\hline Mf I Q / $10 \mathrm{~mm}^{3}$ & $718+/-22$ & $358+/-26$ & $59+/-23$ & $5,34+/-52$ \\
Mf I F / $10 \mathrm{~mm}^{3}$ & $1421+/-11$ & $734+/-12$ & $78+/-18$ & $0,83+/-0,85$ \\
Mf I Q & $\mathrm{R} 69=0,5$ & $\mathrm{R} 70=0,49$ & $\mathrm{R} 71=0,76$ & $\mathrm{R} 72=6,43$ \\
Mf I F & & & & \\
\hline
\end{tabular}

Mf I = nombre moyen de microfilaires ingérées

$\mathrm{Q}=$ queue $; \mathrm{F}=$ flanc.

- Pour $D$. robini et $D$. gracile, le nombre de microfilaires ingérées à la queue est deux fois plus faible qu'au flanc $(\mathrm{R} 69=0,5$ et $\mathrm{R} 70=0,49)$ ce qui correspond exactement aux résultats obtenus dans l'expérience précédente.

- Pour M.(T.) mariae, le nombre de microfilaires ingérées à la queue est également plus faible $(\mathrm{R} 71=0,76)$ mais nettement moins que dans l'expérience précédente : à $21 \mathrm{~h}$ Mf $\mathrm{I} Q / \mathrm{F}=0,28$ et à $9 \mathrm{~h}$ Mf $\mathrm{I} \mathrm{Q} / \mathrm{F}=0,5$.

- Pour $M(T$.$) colombiensis le rapport Q / F$ n'a pas de signification.

\section{D - Variations de l'ingestion lors de la montée ou de la chute de la microfilarémie}

1 - Pendant la montée de la microfilarémie

a) Expérience préliminaire

Les gorgements d'Aedes ont été effectués alors que la microfilarémie est en phase croissante (pic naturel ou pic qui suit l'anesthésie). Elle constitue seulement un test, car d'une part la microfilarémie est mesurée uniquement avant les gorgements, d'autre part un laps de temps important sépare la mesure de la microfilarémie et le gorgement (entre $15^{\prime}$ et $30^{\prime}$ suivant le cas).

Protocole expérimental

— anesthésie du Saïmiri à $21 \mathrm{~h}$ (première anesthésie)

- mesure de la microfilarémie à l'oreille à $21 \mathrm{~h} 05$

- gorgement des Aedes (4 ㅇ) entre 21 h 20 et 21 h 30 
- anesthésie du Saïmiri à $12 \mathrm{~h}$ le lendemain matin (2e anesthésie)

- mesure de la microfilarémie à l'oreille à $12 \mathrm{~h} 05$

- gorgement des Aedes (10 o) entre 12 h 30 et 12 h 45

- anesthésie du Saïmiri à $18 \mathrm{~h}$ ( $3^{\mathrm{e}}$ anesthésie)

- mesure de la microfilarémie à l'oreille à $18 \mathrm{~h} 05$

- gorgement des Aedes (11 o) entre 18 h 25 et 18 h 35.

TABleau XXXVI. - Ingestion des microfilaires de S. sciureus par A. aegypti, en phase de microfilarémie croissante.

Anesthésies à $21 \mathrm{~h}-12 \mathrm{~h}-18 \mathrm{~h}-14-15$ septembre 1983

\begin{tabular}{|c|c|c|c|c|}
\hline & & $21 \mathrm{~h} 20$ & 12 h 30 & 18 h 30 \\
\hline D. robini & $\begin{array}{l}\text { Mf I F / A } \\
\text { Mf I F / } 10 \mathrm{~mm}^{3} \\
\text { Mf O/ } 10 \mathrm{~mm}^{3} \\
\frac{\text { Mf I F }}{\text { Mf O }}\end{array}$ & $\begin{array}{c}627+1-19 \\
1791+-19 \\
787+/-12 \\
\mathrm{R} 73=2,27\end{array}$ & $\begin{array}{c}110+/-14 \\
314+/-14 \\
45+/-14 \\
\mathrm{R} 74=6,97\end{array}$ & $\begin{array}{r}165+/-10 \\
471+-10 \\
44+/-17 \\
\mathrm{R} 75=10,70\end{array}$ \\
\hline D. gracile & $\begin{array}{l}\text { Mf I F / A } \\
\text { Mf I F / } 10 \mathrm{~mm}^{3} \\
\text { Mf O/ } 10 \mathrm{~mm}^{3} \\
\frac{\text { Mf I F }}{\text { Mf O }}\end{array}$ & $\begin{array}{l}314+/-24 \\
897+/-24 \\
201+/-13 \\
R 76=4,46\end{array}$ & $\begin{array}{l}73+1-15 \\
209+/ 15 \\
123+/-13 \\
\mathrm{R} 77=1,70\end{array}$ & $\begin{array}{c}77+/=9 \\
220+-9 \\
119+/=15 \\
\mathrm{R} 78=1,84\end{array}$ \\
\hline$M .(T$.$) mariae$ & $\begin{array}{l}\text { Mf I F / A } \\
\text { Mf I F / } 10 \mathrm{~mm}^{3} \\
\text { Mf O/ } 10 \mathrm{~mm}^{3} \\
\frac{\text { Mf I F }}{\text { Mf O }}\end{array}$ & $\begin{array}{l}92+/-24 \\
263+1-24 \\
177+/-13 \\
\text { R79 }=1,48\end{array}$ & $\begin{array}{c}23+/ 20 \\
66+-20 \\
91+/-13 \\
\mathrm{R} 80=0,72\end{array}$ & $\begin{array}{r}56+/ 15 \\
160+/-15 \\
84+/-16 \\
R 81=1,90\end{array}$ \\
\hline $\begin{array}{l}M .(T .) \\
\text { colombiensis }\end{array}$ & $\begin{array}{l}\text { Mf I F / A } \\
\text { Mf I F / } 10 \mathrm{~mm}^{3} \\
\text { Mf O / } 10 \mathrm{~mm}^{3} \\
\text { Mf I F } \\
\text { Mf O }\end{array}$ & $\begin{array}{c}0,5+1-1,6 \\
1,4+1-4,5 \\
7,7+-36 \\
\mathrm{R} 82=0,2\end{array}$ & $\begin{array}{c}0,5+1-0,5 \\
1,4+/-1,4 \\
1,5+/=164 \\
R 83=0,93\end{array}$ & $\begin{array}{c}0,2+1-0,3 \\
0,6+1-0,9 \\
0 \\
\mathrm{R} 84=-\end{array}$ \\
\hline
\end{tabular}

Mf $\mathrm{I}=$ nombre moyen de microfilaires ingérées

Mf $=$ microfilarémie $; \mathrm{O}=$ oreille $; \mathrm{F}=$ flanc $; \mathrm{A}=$ Aedes.

Le tableau $X X X V I$ représente, pour les 4 espèces de Filaires, le rapport du nombre moyen de microfilaires ingérées au flanc (exprimé pour $10 \mathrm{~mm}^{3}$, avec une estimation de $3,5 \mathrm{~mm}^{3}$ de sang ingéré par Aedes) sur la microfilarémie à l'oreille avant le gorgement.

L'examen de ce tableau montre une série de rapport particulièrement forts qui paraissent se produire en phase de microfilarémie croissante; ce sont les rapports suivants :

pour D. robini $\mathrm{R} 74=6,97(12 \mathrm{~h}), \mathrm{R} 75=10,70(18 \mathrm{~h})$

pour $D$. gracile $\mathrm{R} 76=4,46(21 \mathrm{~h})$

pour $M .(T$.) mariae $\mathrm{R} 81=1,90(18 \mathrm{~h})$.

Ces rapports seront à nouveau considérés chapitre viI. 
b) Expérience de contrôle

L'examen de ces rapports Mf I F/O, très forts en phase de microfilarémie croissante, nous a poussé à réaliser une expérience plus rigoureuse où la microfilarémie à l'oreille est mesurée avant et après le gorgement, avec des intervalles de temps les plus réduits possibles entre la réalisation des prises de sang et le gorgement des moustiques. Nous avons choisi de travailler dans des conditions bien définies, c'est-à-dire lors du pic de microfilarémie de $D$. robini et de $D$. gracile qui suit l'injection d'anesthésique à $9 \mathrm{~h}$ (première anesthésie).

Protocole expérimental :

- anesthésie à $9 \mathrm{~h}$,

- mesure de la microfilarémie à l'oreille avant et après chaque gorgement ( 9 h 12 9 h 22 - 9 h 33 - 9 h 46 - 10 h 01),

- gorgement de 4 lots d'Aedes le premier entre 9 h 14 et 9 h 19 (15 q), le deuxième entre 9 h 24 et 9 h 32 (11 q), le troisième entre 9 h 37 et 9 h 43 (9 o), le dernier entre 9 h 49 et 9 h 52 ( 9 ㅇ) ,

— volume moyen de sang ingéré par chaque Aedes : $2,85 \mathrm{~mm}^{3}$.

Les rapports Mf I F/O sont représentés dans le tableau $X X X V I I$; la valeur de la microfilarémie à l'oreille pendant le gorgement est calculée comme précédemment pour une heure moyenne de gorgement située aux $2 / 3$ entre le début et la fin du repas.

- Pour D. robini, c'est pendant la montée la plus rapide de la phase de microfilarémie croissante que les Aedes ont ingéré en proportion le plus de microfilaires $(\mathrm{R} 86=2,06$ entre $9 \mathrm{~h} 37$ et $9 \mathrm{~h} 43)$; les autres rapports Mf I F/Mf O sont conformes à ceux déterminés lors du pic (R33) ou du creux de microfilarémie (R25).

- Pour $D$. gracile, le rapport le plus fort $(\mathrm{R} 90=1,86)$ est enregistré également pendant la phase aiguë de croissance de la microfilarémie (9 h 49-52), qui est plus tardive que celle de $D$, robini.

- Pour M.(T.) mariae, paradoxalement, les rapports Mf I F/Mf O augmentent régulièrement au cours des 4 gorgements (de 1,35 à 2,44). Cette augmentation est liée non pas à une augmentation du nombre de microfilaires ingérées qui reste stable mais à la chute régulière de la microfilarémie à l'oreille.

- Pour $M$.(T.) colombiensis, les rapports ne montrent pas de phénomène particulier, ils sont tous très faibles en raison de la rareté de cette microfilaire au flanc.

\section{2) Pendant la chute de la microfilarémie}

L'expérience est réalisée à $4 \mathrm{~h}$ solaire, exceptionnellement sans anesthésie, pour éviter une remontée de la microfilarémie de $D$. robini et $D$. gracile, due à l'action de l'anesthésique. 
Tableau XXXVII. - Ingestion des microfilaires de S. sciureus par A. aegypti, en phase de microfilarémie croissante.

Anesthésie à $9 \mathrm{~h}-14$ octobre 1983

\begin{tabular}{|c|c|c|c|c|}
\hline D. robini & 9 h 16 & 9 h 28 & 9 h 40 & 9 h 52 \\
\hline $\begin{array}{l}\text { Mf I F / A } \\
\text { Mf I F / } 10 \mathrm{~mm}^{3} \\
\text { Mf O / } 10 \mathrm{~mm}^{3} \\
\text { Mf I F } \\
\text { Mf O }\end{array}$ & $\begin{array}{c}27+/-11 \\
95+/-11 \\
70+/-19 \\
\mathrm{R} 25=1,38\end{array}$ & $\begin{array}{l}75+/-18 \\
263+/-18 \\
170+/-15 \\
\mathrm{R} 85=1,54\end{array}$ & $\begin{array}{c}342+/-12 \\
1200+/-12 \\
580+/-14 \\
\mathrm{R} 86=2,06\end{array}$ & $\begin{array}{c}431+/-7 \\
1512+/-7 \\
920+/-12 \\
\mathrm{R} 87=1,64\end{array}$ \\
\hline D. gracile & 9 h 16 & 9 h 28 & 9 h 40 & 9 h 52 \\
\hline $\begin{array}{l}\text { Mf I F / A } \\
\text { Mf I F / } 10 \mathrm{~mm}^{3} \\
\text { Mf O / } 10 \mathrm{~mm}^{3} \\
\text { Mf I F } \\
\text { Mf O }\end{array}$ & $\begin{array}{l}54+/-14 \\
189+/-14 \\
125+/-18 \\
\mathrm{R} 26=1,45\end{array}$ & $\begin{array}{l}68+/-12 \\
239+/-12 \\
148+/-14 \\
\mathrm{R} 88=1,61\end{array}$ & $\begin{array}{c}103+/-12 \\
361+/-12 \\
235+/-14 \\
\mathrm{R} 89=1,53\end{array}$ & $\begin{array}{c}205+/-7 \\
719+/-7 \\
400+/-12 \\
\mathrm{R} 90=1,86\end{array}$ \\
\hline$M$. (T.) mariae & 9 h 16 & 9 h 28 & $9 \mathrm{~h} 40$ & 9 h 52 \\
\hline $\begin{array}{l}\text { Mf I F / A } \\
\text { Mf I F / } 10 \mathrm{~mm}^{3} \\
\text { Mf O / } 10 \mathrm{~mm}^{3} \\
\frac{\text { Mf I F }}{\text { Mf O }}\end{array}$ & $\begin{array}{r}33+/-10 \\
116+/-10 \\
90+/-18 \\
\mathrm{R} 27=1,35\end{array}$ & $\begin{array}{r}40+/-17 \\
140+/-17 \\
85+/-15 \\
\mathrm{R} 91=1,65\end{array}$ & $\begin{array}{l}41+/-15 \\
144+/-15 \\
70+/-15 \\
\mathrm{R} 92=2,05\end{array}$ & $\begin{array}{r}35+/-13 \\
122+/-13 \\
50+/-14 \\
\mathrm{R} 93=2,44\end{array}$ \\
\hline $\begin{array}{l}M .(T .) \\
\text { colombiensis }\end{array}$ & 9 h 16 & 9 h 28 & 9 h 40 & 9 h 52 \\
\hline $\begin{array}{l}\text { Mf I F / A } \\
\text { Mf I F / } 10 \mathrm{~mm}^{3} \\
\text { Mf O / } 10 \mathrm{~mm}^{3} \\
\frac{\text { Mf I F }}{\text { Mf O }}\end{array}$ & $\begin{array}{c}0,26+/-0,34 \\
0,91+/-1,2 \\
19+/-41 \\
\mathrm{R} 28=0,05\end{array}$ & $\begin{array}{c}0,63+/-0,34 \\
2,21+/-1,19 \\
23+/-33 \\
\mathrm{R} 94=0,1\end{array}$ & $\begin{array}{c}0,67+/-0,66 \\
2,35+/-2,31 \\
22+/-33 \\
\mathrm{R} 95=0,11\end{array}$ & $\begin{array}{c}0,11+/-0,26 \\
0,44+/-1,07 \\
13+/-30 \\
\mathrm{R} 96=0,03\end{array}$ \\
\hline
\end{tabular}

Mf $\mathrm{I}=$ nombre moyen de microfilaires ingérées

Mff $=$ microfilarémie $; \mathrm{O}=$ oreille $; \mathrm{F}=$ flanc $; \mathrm{A}=$ Aedes.

Protocole expérimental :

- mesure de la microfilarémie à l'oreille avant et après le repas ( $3 \mathrm{~h} 58$ et $4 \mathrm{~h} 13$ )

- gorgement des moustiques sur le flanc entre 4 h et 4 h 10 ; le singe est immobilisé dans un grillage plastique souple.

- volume moyen de sang ingéré par Aedes $=2,14 \mathrm{~mm}^{3}$. 
L'évolution des densités microfilariennes entre les deux prises de sang à l'oreille est la suivante :

$$
\begin{array}{lr}
\text { D. robini } & 441-143 \\
\text { D. gracile } & 691-623 \\
M .(T .) \text { mariae } & 166-53 \\
M .(T .) \text { colombiensis } & 71-52
\end{array}
$$

La chute de la microfilarémie est faible pour $D$. gracile mais à 4 h cette filaire est pratiquement encore au pic ( $f i g .7$ ).

Le tableau XXXVIII représente les rapports Mf I F/O (pour $10 \mathrm{~mm}^{3}$ ), calculés pour une heure moyenne de gorgement aux $2 / 3$ du temps entre 3 h 58 et 4 h 13 .

TABLEAU XXXVIII. - Ingestion des microfilaires de S. sciureus par A. aegypti, en phase de chute de la microfilarémie.

Pas d'anesthésie - 5 novembre 1983

\begin{tabular}{lrrrc}
\hline & D. robini & D. gracile & $M .(T$.$) mariae$ & $\begin{array}{c}M .(T .) \\
\text { colombiensis }\end{array}$ \\
\hline Mf I F / A & $82+/-20$ & $171+/-17$ & $38+/-26$ & $0,6+/-0,6$ \\
Mf I F / 10 mm & $383+/-20$ & $799+/-17$ & $178+/-26$ & $2,8+/-2,8$ \\
Mf O / 10 mm & $243+/-12$ & $646+/-10$ & $90+/-14$ & $52+/-27$ \\
Mf I F & $\mathrm{R} 97=1,58$ & $\mathrm{R} 98=1,24$ & $\mathrm{R} 99=1,96$ & $\mathrm{R} 100=0,05$ \\
Mf O & & & & \\
\hline
\end{tabular}

Mf $\mathrm{I}=$ nombre moyen de microfilaires ingérées

Mf $=$ microfilarémie

$\mathrm{O}=$ oreille $; \mathrm{F}=$ flanc $; \mathrm{A}=$ Aedes

Les rapports déterminés ici seront analysés de nouveau (chap. vII), en particulier celui de $M .(T$.) mariae qui est très fort $(\mathrm{R} 99=1,96)$.

\section{E - Distributions des vecteurs en fonction du nombre de microfilaires ingérées}

\section{1 - Aedes aegypti}

La distribution des vecteurs en fonction du nombre de microfilaires ingérées est prise en considération par les épidémiologistes car c'est un des facteurs les plus importants dans la stratégie de la transmission des parasites (Anderson, 1978; Schmidt et coll., 1972).

Dans ce travail, le coefficient $\mathrm{K}$ de Neymann (chap. I) nous donne l'allure générale de la distribution et nous permet de juger de la répartition homogène ou agglomérée des 4 espèces de microfilaires dans leurs biotopes respectifs.

Pour l'analyse des distributions nous utilisons une expérience faite avec $50 \mathrm{~A}$ edes gorgés à réplétion. 
Les repas se sont effectués durant les $15^{\prime}$ qui suivent l'anesthésie du singe, faite à $9 \mathrm{~h}$, pour que l'hétérogénéité due à des variations de la microfilarémie ne soit pas trop grande.

Tableau XXXIX. - Les Filaires de $S$. sciureus; distribution des Aedes en fonction du nombre de microfilaires ingérées au flanc.

Anesthésie à $9 \mathrm{~h}-31$ janvier 1983

\begin{tabular}{|c|c|c|c|c|}
\hline & D. robini & D. gracile & $M$. (T.) mariae & $\begin{array}{c}M .(T .) \\
\text { colombiensis }\end{array}$ \\
\hline $\begin{array}{l}\text { Mf I F / A } \\
\text { EXT } \\
\text { SX }^{2} \\
\text { K }\end{array}$ & $\begin{array}{c}25+1-5 \\
7-44 \\
67 \\
14,88\end{array}$ & $\begin{array}{c}54+/-5 \\
24 \frac{104}{403} \\
8,36\end{array}$ & $\begin{array}{c}153+/-5 \\
32-303 \\
3753 \\
6,50\end{array}$ & $\begin{array}{c}1,02+1-0,28 \\
0 \frac{-4}{0,98} \\
-\end{array}$ \\
\hline
\end{tabular}

$$
\begin{array}{ll}
\text { Mf I F / A } & =\text { nombre moyen de microfilaires ingérées au flanc par Aedes } \\
\mathrm{EXT} & =\text { nombres extrêmes de microfilaires ingérées } \\
\mathrm{K} & =\text { coefficient d'aggrégation de Neymann } \\
\mathrm{S}^{2} & =\text { variance } \\
A & =\text { Aedes }
\end{array}
$$

- La forte valeur de $\mathrm{K}$ pour $D$. robini montre que cette Filaire est distribuée d'une façon très homogène dans le sang cutané accessible à l'Aedes.

- D. gracile est également distribué d'une façon homogène mais moins que D. robini.

- M.(T.) mariae est la microfilaire qui a le plus tendance à former des aggrégats $(\mathrm{K}=6,5)$ même si son coefficient d'aggrégation est encore élevé. Cette tendance est illustrée également par la grande différence entre les valeurs extrêmes observées.

- Pour M.(T.) colombiensis, la moyenne est basse et les méthodes de calcul de K ne donnent pas de solution satisfaisante. Cependant la distribution peut s'ajuster à une loi de Poisson car le rapport Variance/Moyenne ne diffère pas significativement de 1 (Test de Bliss et Fisher, 1953).

Cette microfilaire paraît donc répartie d'une façon régulière dans le sang du flanc.

\section{2 - Culicoïdes nubeculosus}

Chaque Aedes ingère de 3 à $5 \mathrm{~mm}^{3}$ de sang. Il est clair lorsqu'on assiste au repas (chap. III) qu'il s'agit dans la plupart des cas de la somme de plusieurs types de repas.

Culicoïdes nubeculosus qui n'ingère en moyenne que $0,3 \mathrm{~mm}^{3}$ de sang doit nous permettre une analyse plus fine et nous montrer en particulier l'existence éventuelle des concentrations des microfilaires dans certains vaisseaux.

Les cinq expériences réalisées (2 en nourrissant les Culicoïdes sur le flanc et 3 en les nourrissant sur l'oreille) sont exposées dans le tableau XL. 
Tableau XL. - Ingestion des microfilaires de S. sciureus par C. nubeculosus.

\begin{tabular}{|c|c|c|c|c|c|}
\hline & D. robini & D. gracile & $\begin{array}{l}M .(T .) \\
\text { mariae }\end{array}$ & $\begin{array}{c}M .(T .) \\
\text { colombiensis }\end{array}$ & Exp. no \\
\hline Mf I F/C & $35 \pm 18$ & $13 \pm 18$ & $6 \pm 22$ & 0 & 1 \\
\hline EXT. & $0 \div 155$ & $1 \div 65$ & $0=34$ & - & \\
\hline $\begin{array}{l}\mathrm{K} \\
\mathrm{Mf} I \mathrm{~F} / \mathrm{C}\end{array}$ & $\underline{0,90}$ & $5^{0,89}+10$ & $7+11$ & $\overline{0}$ & 2 \\
\hline EXT. & - & $0 \simeq 48$ & $0 \simeq 78$ & 0 & \\
\hline $\mathrm{K}$ & - & 2,28 & 1,61 & - & \\
\hline Mf I OD/C & $1,5 \pm 36$ & $10 \pm 34$ & $8 \pm 34$ & 0 & 3 \\
\hline EXT. & $0 \div 7$ & $0 \div 38$ & $0=70$ & - & \\
\hline Mf I OV/C & $10 \pm 24$ & $6+26$ & $8+25$ & 0 & 4 \\
\hline EXT. & $0=20$ & $2-27$ & $0-20$ & - & \\
\hline Mf I OV/C & $34 \pm 30$ & $25 \pm 28$ & $1,4 \pm 30$ & 0,04 & 5 \\
\hline EXT. & $0=211$ & $0-148$ & $0-8$ & $0-1$ & \\
\hline
\end{tabular}

Exp. no $1: 83$ Culicoîdes gorgés sur le flanc — anesthésie à $9 \mathrm{~h}$, le 21 janvier 1983.

$n^{\circ} 2$ : 58 Culicoïdes gorgés sur le flanc - anesthésie à $9 \mathrm{~h}$, le 22 mars 1983 (expérience réalisée exceptionnellement sur un deuxième singe, qui a les mêmes espèces de microfilaires sauf $D$. robini).

no $3: 13$ Culicoïdes gorgés sur la face dorsale de l'oreille - anesthésie à $9 \mathrm{~h}$, le 2 août 1983 .

no 4 : 10 Culicoïdes gorgés sur la face ventrale de l'oreille - anesthésie à $9 \mathrm{~h}$, le 2 août 1983.

no $5: 24$ Culicoïdes gorgés sur la face ventrale de l'oreille à 10 h 30 après anesthésie à $9 \mathrm{~h}$, le 3 novembre 1983 .

Mf I OD : nombre de microfilaires ingérées sur la partie dorsale de l'oreille.

Mf I OV : idem sur la partie ventrale.

Mf I F : nombre de microfilaires ingérées sur le flanc.

$\mathrm{C}$ : Culicoïde.

K : coefficient d'aggrégation de Neymann.

EXT : nombres extrêmes de microfilaires ingérées.

On peut en extraire les notions suivantes :

1) Dans les 2 expériences où le coefficient $\mathrm{K}$ peut être calculé précisément (échantillon suffisant), nous constatons à nouveau que $M$.(T.) mariae a une distribution plus dispersée que $D$. robini et $D$. gracile.

2) Contrairement aux Aedes, la distribution n'est plus proche du modèle poissonien $(\mathrm{K}>7)$ mais est très surdispersée $(\mathrm{K}<3)$.

3) Les culicö̈des qui constituent les cas extrêmes sont intéressants. Dans l'expérience Oreille no 3 , c'est le même culicoïde, gorgé sur la face dorsale de l'oreille, qui a ingéré 70 microfilaires de $M$.(T.) mariae, 38 microfilaires de $D$. gracile et 7 microfilaires de D. robini. Le nombre le plus élevé de microfilaires ingérées pour les 3 espèces est donc constaté chez le même culicoïdes.

Dans l'expérience Oreille $\mathrm{n}^{0}$ 5, le nombre de microfilaires de $M$.(T.) mariae ingérées est très faible, mais à cette époque la microfilarémie de cette Filaire est devenue très basse. 
Il apparaît donc nettement que lorsqu'une espèce est anormalement abondante dans le repas d'un culicoïde, les 2 autres espèces sont elles aussi anormalement abondantes dans le même prélèvement. Cela évoque l'existence de microniches où se concentrent les microfilaires quelque soit l'espèce.

4) Une seule microfilaire de $M .(T$.) colombiensis a été ingérée par les 48 culicoïdes gorgés sur l'oreille ( 3 exp.) alors que cette Filaire est très fréquente en histologie au niveau des capillaires superficiels de l'oreille.

La biologie très particulière de cette microfilaire est confirmée ici ; elle paraît coincée dans les capillaires sous-épidermiques, les Aedes et même les culicoïdes ne pouvant la "décrocher " que difficilement.

\section{Chapitre VII : Synthèse des données}

Certains faits d'importance pratique apparaissent immédiatement dans les chapitres précédents :

- l'ingestion par l'Aedes au flanc est très supérieure à ce que prélève l'expérimentateur à l'oreille pour trois des Filaires (D. robini, D. gracile, $M$. (T.) mariae) et, au contraire très inférieure pour la quatrième espèce ( $M$. (T.) colombiensis).

- Lorsque l'expérimentateur prélève du sang à la pointe de la queue par les méthodes habituelles, il surestime considérablement le taux de la microfilarémie.

Si ces faits apparaissent donc sans aucune ambiguité, les mécanismes de l'ingestion sont difficiles à démonter. Nous allons tenter de le faire dans ce chapitre.

En combinant l'ensemble des données disponibles nous allons envisager successivement la localisation la plus vraisemblable de chacune des 4 espèces de microfilaires dans l'arbre vasculaire, puis les particularités vasculaires des 3 organes ponctionnés (oreille, flanc, queue). Cela va permettre de formuler des hypothèses concernant : 1) la composition de chaque prélèvement (expérimentateur ou Aedes) en sang provenant de petits vaisseaux (moins de $15 \mu \mathrm{m}$ ), moyens vaisseaux (15 à $70 \mu \mathrm{m}$ ) et gros vaisseaux (plus de $70 \mu \mathrm{m})$; 2) la richesse respective de ces 3 catégories de vaisseaux en chacune des 4 espèces de microfilaires.

Ces hypothèses impliquent des conséquences quantitatives qui seront testées à l'aide de la centaine de rapports établis expérimentalement.

\section{A - Particularités dans le comportement et la localisation des microfilaires de chacune des quatre espèces}

\section{$1-M .(T$.$) colombiensis$}

A frais, cette microfilaire apparaît robuste et assez rigide; elle est peu mobile et avance lentement. La simple analyse histologique a montré que $M$. (T.) colombiensis a un comportement et une localisation très spéciaux. Son corps, qui atteint $6 \mu \mathrm{m}$ de diamètre, emplit littéralement un grand nombre de capillaires. 
Paradoxalement c'est donc l'espèce la plus épaisse qui fréquente la partie la plus fine du réseau vasculaire. Nous verrons qu'elle est dans une certaine mesure immobilisée dans cette situation et que la proportion de la population qui circule normalement dans le sang est faible.

\section{$2-M .(T$.$) mariae$}

A frais, cette microfilaire est très souple, grêle, et se pelotonne sur elle-même ; elle n'avance pas.

Nous n'avons pas de preuves directes de la fréquentation microvasculaire de $M$.(T.) mariae. Plusieurs arguments font penser cependant que, par l'enroulement de son corps sur elle-même, elle se maintient dans de fins vaisseaux mieux que les deux Dipetalonema qui ont pourtant un diamètre du corps supérieur. Les arguments sont essentiellement :

a) le phénomène de la première goutte de sang prélevée à la queue. La " première goutte ", de très faible volume, contient principalement des hématies provenant des capillaires. Elle est extraordinairement riche en microfilaires de $M .(T$.) mariae (chap. v, Bl).

b) les prises de sang répétées sur 24 heures où $M .(T$.$) mariae a un compor-$ tement qui rappelle celui de $M$.(T.) colombiensis (chap. v, B2).

c) la répartition très hétérogène de $M$.(T.) mariae dans les repas des Culicoides (chap. vI, E2), comme si ceux-ci tombaient sur des " nids " de microfilaires de cette espèce ; celles-ci, retenues dans les fins vaisseaux et moins bien brassées dans le courant sanguin auraient une répartition plus contagieuse; elles formeraient des bouchons transitoires capables de freiner $D$, robini et $D$. gracile.

\section{3 - D. gracile et D. robini}

A frais, $D$. gracile se déplace par ondulations, elle est peu puissante et bien mobile. $D$. robini se déplace par ondulations puissantes, elle apparaît comme la microfilaire la plus rapide.

Les deux Dipetalonema ont un comportement périodique très marqué et correspondent mieux à ce qui est admis classiquement dans la biologie des microfilaires.

Nous n'avons aucune preuve directe ou indirecte de leur présence dans les fins vaisseaux.

Il s'agit donc de microfilaires plus passives qui s'accrochent peu aux parois et sont brassées à grande vitesse dans le torrent circulatoire en même temps que les hématies. Il en résulte que, mieux que pour les deux espèces précédentes, les rapports expérimentaux obtenus sont conformes à ce que l'on peut attendre sachant que D. robini a une périodicité plus forte (rapport d'environ 20 au lieu de 8 entre le taux le plus bas et le taux le plus élevé) et plus précoce $(21 \mathrm{~h}-0 \mathrm{~h}$ au lieu de $0 \mathrm{~h}-3 \mathrm{~h}$ ).

La taille et la musculature plus puissante de $D$. robini pourraient cependant apporter des nuances dans le comportement des deux espèces. 
Si l'on tient compte du fait qu'un réseau vasculaire se dichotomise de façon assez régulière, il arrive un moment où les microfilaires qui ne pénètrent pas dans les très fins vaisseaux, ne peuvent suivre les hématies et doivent trouver un trajet d'anastomose pour boucler leur périple.

D. gracile, plus passive, paraît le faire sans difficulté. D. robini, au contraire, pourrait à ce moment se trouver freinée provisoirement dans des vaisseaux de moyen calibre et cette accumulation passagère fournirait une explication à différents rapports dans lesquels les valeurs pour $D$. robini et $D$. gracile sont clairement différentes.

\section{B - Particularités vasculaires des différents organes pouvant fournir une expli- cation aux résultats expérimentaux}

Si nous reprenons les résultats des analyses histologiques faites précédemment, nous pouvons schématiser les caractéristiques des trois organes en disant :

- Oreille : nombreux capillaires, une artère et une veine marginale de grand diamètre (400 à $500 \mu \mathrm{m})$, des vaisseaux de 20 à $30 \mu \mathrm{m}$ de diamètre.

- Flanc : peu de capillaires, peu de gros vaisseaux (300 $\mu \mathrm{m})$, abondance d'un réseau richement anastomosé d'un diamètre avoisinant $70 \mu \mathrm{m}$.

- Queue : la queue paraît particulièrement pauvre en vaisseaux de moyen calibre. A l'extrémité elle est très riche en capillaires. Plus antérieurement, elle possède le long des vertèbres de gros vaisseaux à musculature puissante et de nombreux glomi ; les fins vaisseaux deviennent rares.

Spontanément, le sang vient difficilement à l'extrémité de la queue et l'on est astreint à effectuer un massage d'avant en arrière ce qui comprime les vaisseaux contre les vertèbres; le sang vient alors régulièrement et en abondance suffisante.

Ces particularités ont des conséquences nettes sur la richesse du sang en microfilaires. La première goutte de sang prise à l'extrémité est étonnamment riche en parasites localisés dans les très fins vaisseaux. La suite du prélèvement est moins riche; il semble même que l'on puisse parler d'un phénomène de vidage car les parasites qui peuvent être extraits des fins vaisseaux par ces procédés ne peuvent se renouveler en quelques secondes. Par contre, lorsque les prises de sang sont effectuées pendant par exemple toute une journée, avec quelques heures d'intervalle entre ces prises de sang, nous constatons un enrichissement relatif des derniers prélèvements dû vraisemblablement à la congestion générale de la queue et à la dilatation des petits vaisseaux.

L'ensemble de ces phénomènes est donc très complexe et c'est finalement la piqûure de l'Aedes qui, en supprimant la plupart de ces artefacts, fournit les données les plus constantes sur la densité des microfilaires à la queue. 


\section{C - Hypothèses sur les particularités des sangs prélevés par l'expérimentateur ou par l'Aedes et sur les particularités des répartitions de chaque espèce de microfilaires dans les vaisseaux}

L'ensemble des considérations précédentes nous indique qu'il est nécessaire pour analyser les résultats de définir schématiquement trois sortes de vaisseaux :

a) Les PETITS vaIsseaux dans lesquels se trouvent $M .(T$.) colombiensis et où vraisemblablement $M$.(T.) mariae est susceptible de séjourner mais que les Dipetalonema gracile et robini ne fréquentent pas. Il s'agit de tous les vaisseaux artériels, veineux ou capillaires ayant un diamètre égal ou inférieur à $15 \mu \mathrm{m}$.

b) Les vaisseaux DE MOYEn DiAmÈTRE qui constituent le réseau richement anastomosé essentiellement situé dans le tissu adipeux sous-cutané, parallèlement à la surface de la peau. Pour fixer les idées nous estimerons que le diamètre de ces vaisseaux est de l'ordre de 15 à $70 \mu \mathrm{m}$.

c) Les vaisseaux DE GRos DiAmètre où les microfilaires, comme les hématies, circulent librement à très grande vitesse. Le diamètre de ces artères ou veines est donc estimé égal ou supérieur à $70 \mu \mathrm{m}$.

Pour préciser les particularités inhérentes à chaque espèce lors de l'ingestion par le vecteur, il faut tenter tout d'abord de préciser l'abondance relative des quatre espèces dans ces trois catégories de vaisseaux.

Il est apparu que les appréciations directes sont très aléatoires car, en plus de la périodicité propre de chacune des Filaires, le moindre stress de l'hôte modifie très rapidement et de façon importante les densités microfilariennes.

Il en résulte qu'on ne peut comparer les expériences de piqûre par Aedes, qui durent nécessairement plusieurs minutes, et les ponctions de sang faites par l'expérimentateur.

Les seuls résultats que nous utilisons comme base sont donc les taux de chacune des espèces, dans les différents organes pris à quelques instants d'intervalle ; en pratique il s'agit de la comparaison oreille-flanc, la queue se révélant trop complexe.

En effet, si l'on ne tient pas compte de $M$.(T.) colombiensis, où les microfilaires circulantes sont très rares, les valeurs des trois autres espèces sont élevées et ont un faible intervalle de confiance. Les trois espèces coexistant dans la même goutte de sang, les taux relatifs de chacune d'elles sont fiables. M.(T.) colombiensis sera traitée séparément dans le sous-chapitre F.

Nous allons donc, dans un premier temps, nous appuyer sur toute l'expérimentation qui consiste à comparer les prises faites au flanc aux prises faites à l'oreille. Nous allons tenter ( fig. 14):

a) de fixer pour chaque prélèvement la part respective du sang qui provient des vaisseaux de moins de $15 \mu \mathrm{m}$, de 15 à $70 \mu \mathrm{m}$ et de plus de $70 \mu \mathrm{m}$ de diamètre.

b) d'attribuer, en fonction des considérations citées plus haut, un emplacement préférentiel pour chacune des trois espèces de microfilaires circulantes dans chacun des trois types de vaisseaux considérés. 

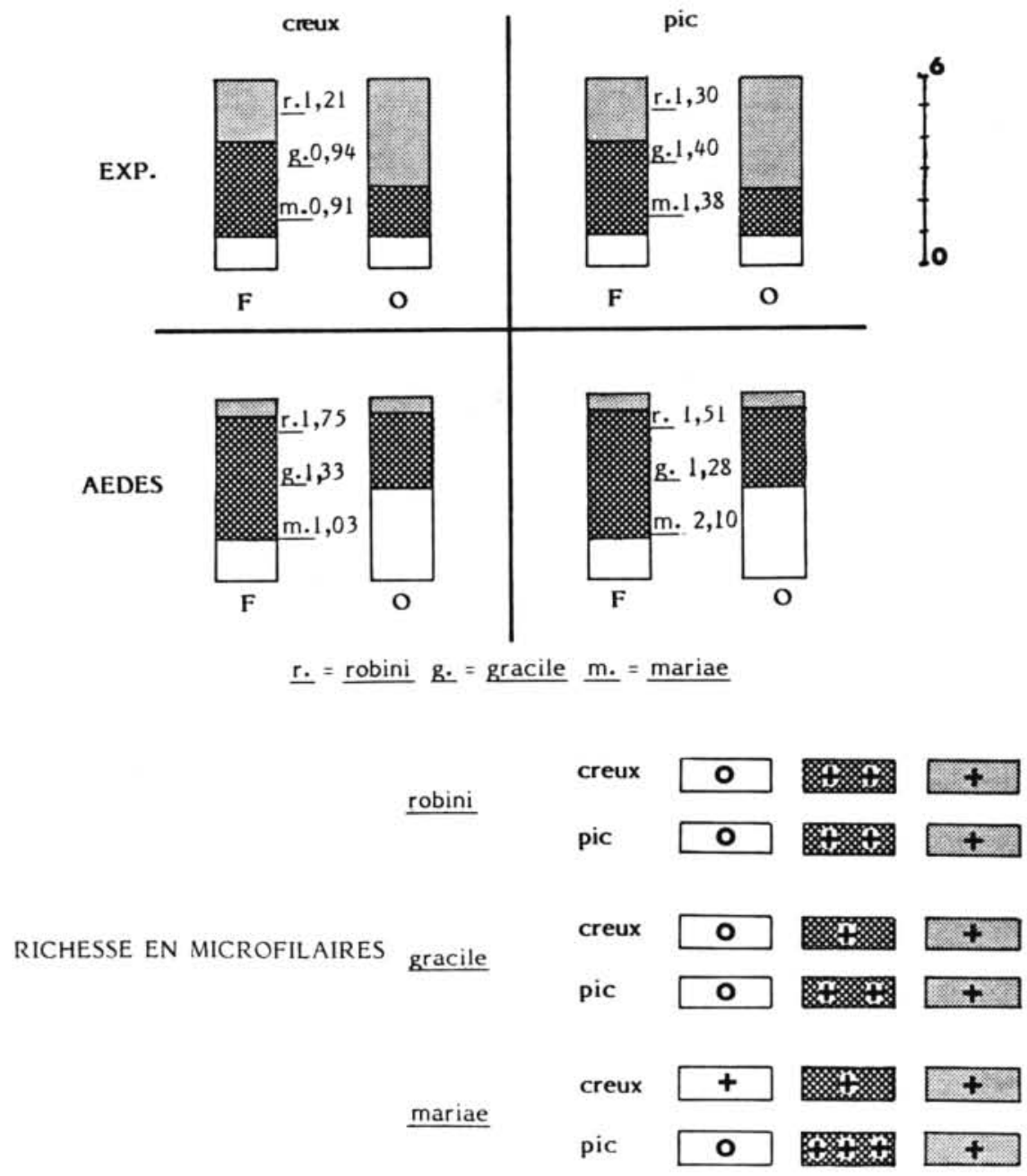

FIg. I 4. - Hypothèses pour rendre compte des I2 rapports flanc/oreille : - en haut à gauche, on suppose qu'un prélèvement de sang est constitué d'hématies provenant en partie de gros vaisseaux de plus de $70 \mu \mathrm{m}$ de diamètre (grisé fin), de moyens vaisseaux de $I_{5}$ à $70 \mu \mathrm{m}$ de diamètre (grisé épais) et de petits vaisseaux de moins de I 5 um de diamètre (blanc). - en bas $\grave{a}$ droite, on suppose que la richesse relative de chacune des trois filaires dans le sang varie selon le diamètre du vaisseau et selon que la microfilarémie est au pic ou au creux. Exemple : $D$. gracile, au moment du pic : pas de microfilaires dans les petits vaisseaux, nombreuses microflaires dans les moyens vaisseaux, abondance moyenne dans les gros vaisseaux. - au centre, la composition hypothétique de chaque prélèvement en sang provenant de gros, moyens ou petits vaisseaux est représentée sur chaque colonne (chaque colonne est divisée en 6 unités, échelle à droite). F : flanc ; $O$ : oreille ; en haut prélèvement fait par l'expérimentateur, en bas prélèvement fait par l'Aedes, à gauche au creux de la microfilarémie, à droite au pic de la miciofilarémie. Entre les 2 colonnes : rapports flanc/oreille pour $D$. robini (r.), D. gracile (g.) et $M$. (T. ( mariae (m.).

Le tableau XXXXI montre la concordance entre les hypothèses formulées et les chiffres obtenus expérimentalement. 
c) Nous vérifierons (tableau XLI) qu'en combinant ces deux hypothèses il sera possible de rendre compte des 12 rapports obtenus expérimentalement dans les comparaisons flanc-oreille. Il sera possible du même coup de vérifier que les astreintes fournies par ces rapports sont suffisamment fortes pour que les hypothèses adoptées en a) d'une part et en b) d'autre part soient pratiquement obligatoires, ce qui démontre, semble-t-il, leur forte vraisemblance.

\section{1 - Hypothèses sur l'origine des sangs prélevés (fig. 14, au centre) \\ - Expérimentateur-flanc :}

Une incision est faite à la lame de rasoir sur une profondeur de $2 \mathrm{~mm}$ environ. Étant donné les indications fournies par l'histologie, nous pensons que le sang prélevé provient en large part des vaisseaux de diamètre moyen. Nous prenons donc comme hypothèse en divisant le sang prélevé en six unités égales:

$$
\begin{array}{rrrr}
\text { sang venant des vaisseaux de moins de } 15 \mu \mathrm{m}: 1 & \text { unité } \\
- & 15 \text { à } 70 \mu \mathrm{m}: 3 & \text { unités } \\
- & - & \text { plus de } 70 \mu \mathrm{m}: 2 & \text { unités }
\end{array}
$$

\section{- Expérimentateur-oreille :}

L'oreille est piquée près du bord et ne saigne correctement que lorsque la veine marginale est atteinte. Nous prenons donc comme hypothèse :

sang venant des vaisseaux de moins de $15 \mu \mathrm{m}: 1$ unité

$$
15 \text { à } 70 \mu \mathrm{m}: 1,5 \text { unité }
$$

plus de $70 \mu \mathrm{m}: 3,5$ unités

- Aedes-flanc :

D'après les observations faites à frais sur la piqûre de l'Aedes et sur l'histologie, il semble bien que l'Aedes ne pique qu'exceptionnellement dans les gros vaisseaux et qu'en moyenne la plupart des repas sur le flanc s'effectuent essentiellement à partir du réseau sous-cutané. Nous proposons donc la répartition suivante :

sang venant des vaisseaux de moins de $15 \mu \mathrm{m}: 1,5$ unité

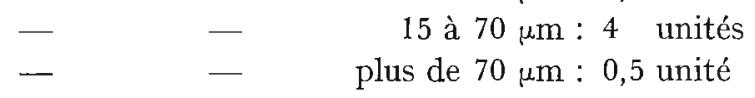

- Aedes-oreille :

Peu d'Aedes atteindront les gros vaisseaux, ils se nourrissent vraisemblablement sur le réseau des petits vaisseaux de moins de $15 \mu \mathrm{m}$. La répartition proposée est donc la suivante :

sang venant des vaisseaux de moins de $15 \mu \mathrm{m}: 3$ unités

$$
\begin{array}{llr}
- & - & 15 \text { à } 70 \mu \mathrm{m}: 2,5 \text { unités } \\
- & - & \text { plus de } 70 \mu \mathrm{m}: 0,5 \text { unité }
\end{array}
$$


2 - Hypothèses sur l'emplacement préférentiel de chacune des trois espèces de microfilaires circulantes ( $f i g .14$ en bas à droite)

En raison des arguments développés plus haut, nous proposons la répartition suivante :

\section{- D. robini}

Une concentration est supposée dans les vaisseaux de 15 à $70 \mu \mathrm{m}$. On peut imaginer en effet que les microfilaires qui arrivent en grande abondance et à grande vitesse par les gros vaisseaux aient une certaine difficulté à poursuivre leur trajet lorsqu'elles parviennent à une dichotomie vasculaire dans laquelle elles ne peuvent pénétrer, ce qui peut entraîner une accumulation non négligeable en aval.

Le phénomène doit être particulièrement marqué pour $D$. robini, la plus robuste et la plus rigide des trois espèces. Nous proposons donc comme formules :

- D. robini — creux de la microfilarémie

moins de $15 \mu \mathrm{m} \quad 0$

15 à $70 \mu \mathrm{m}++$

plus de $70 \mu \mathrm{m}+$

- pic de la microfilarémie

moins de $15 \mu \mathrm{m} \quad 0$

15 à $70 \mu \mathrm{m}+$

plus de $70 \mu \mathrm{m}+$

- D. gracile

Nous adoptons les mêmes hypothèses que pour $D$. robini, mais l'espèce étant plus petite, on peut supposer qu'elle circule plus aisément que $D$. robini et que son accumulation devant les dichotomies vasculaires terminales n'est sensible qu'au moment du pic, lorsque les microfilaires quittent en grande quantité les poumons.

L'hypothèse adoptée est :

- D. gracile - creux de la microfilarémie moins de $15 \mu \mathrm{m} \quad 0$

15 à $70 \mu \mathrm{m}+$ plus de $70 \mu \mathrm{m}+$

- pic de la microfilarémie moins de $15 \mu \mathrm{m} \quad 0$

15 à $70 \mu \mathrm{m}++$ plus de $70 \mu \mathrm{m}+$

- M.(T.) mariae

Le raisonnement suivi pour $D$. gracile s'applique à $M$. (T.) mariae avec pour seule différence la présence supposée de l'espèce dans les vaisseaux de plus fin calibre. La formule proposée sera donc : 
- M.(T.) mariae - creux de la microfilarémie moins de $15 \mu \mathrm{m}+$

15 à $70 \mu \mathrm{m}+$ plus de $70 \mu \mathrm{m}+$

— pic de la microfilarémie moins de $15 \mu \mathrm{m} \quad 0$

15 à $70 \mu \mathrm{m}+++$ plus de $70 \mu \mathrm{m}+$

\section{D - Examen des rapports Flanc/Oreille à l'aide des hypothèses précédentes}

Les données sont regroupées sur la figure 14 et le tablean XLI.

\section{1 - Expérimentateur — creux de microfilarémie}

Pour $D$. robini, le rapport $\mathrm{R} 1$ est égal à 1,21 puisque le sang des vaisseaux de 15 à $70 \mu \mathrm{m}$ (le plus riche en microfilaires) est deux fois plus abondant dans le flanc que dans l'oreille.

Pour $D$. gracile le rapport R2 est proche de 1 car, pendant le creux, les microfilaires sont aussi abondantes dans le sang des vaisseaux de 15 à $70 \mu \mathrm{m}$ que dans celui des vaisseaux de plus de $70 \mu \mathrm{m}$.

Pour $M$. (T.) mariae, le rapport R $3=0,91$ est aussi près de 1 puisque le sang des vaisseaux de moins de $15 \mu \mathrm{m}$, de 15 à $70 \mu \mathrm{m}$ et de plus de $70 \mu \mathrm{m}$ est également riche.

\section{2 - Expérimentateur - pic de microfilarémie}

Les microfilaires de $D$. robini $(\mathrm{R} 5=1,30), D$. gracile $(\mathrm{R} 6=1,40)$ et $M .(T$. mariae $(\mathrm{R} 7=1,38)$ sont toutes les trois plus abondantes dans le flanc que dans l'oreille puisque dans les trois cas, au moment du pic, elles se concentrent dans les vaisseaux de 15 à $70 \mu \mathrm{m}$.

\section{3 - Aedes - creux de microfilarémie}

D. robini ( $42=1,75$ ) est plus riehe dans le flanc puisque le sang des vaisseaux de 15 à $70 \mu \mathrm{m}$ est présumé deux fois plus abondant que dans l'oreille et qu'il est le plus riche en microfilaires.

Ce rapport pour $D$. gracile (R50 = 1,33) est également supérieur à 1 pour les mêmes raisons que pour $D$. robini mais plus faiblement car la richesse en microfilaires de $D$. gracile dans les vaisseaux de 15 à $70 \mu \mathrm{m}$ est relativement moins forte.

Pour $M .(T$.) mariae (R58 = 1,03), le rapport est égal à 1 puisque la richesse en microfilaires dans les vaisseaux de moins de $15 \mu \mathrm{m}$ et dans les vaisseaux de 15 à $70 \mu \mathrm{m}$ est comparable.

\section{4 - Aedes - pic de microfilarémie}

Les rapports pour D. robini $(\mathrm{R} 38=1,51)$, D. gracile $(\mathrm{R} 46=1,28)$ et $M .(T$. mariae (R54 $=2,1$ ) sont tous les trois bien supérieurs à 1 du fait que dans les trois cas, les microfilaires sont plus abondantes, ou beaucoup plus abondantes, dans les vaisseaux de 15 à $70 \mu \mathrm{m}$ que dans ceux de moins de $15 \mu \mathrm{m}$. 
TABLEAU XLI. - Rapports Flanc/Oreille des prélèvements de sang faits par l'expérimentateur (EXP.) d'une part, et par l'Aedes d'autre part ; comparaison des valeurs obtenues dans l'expérience de 20-21 juin 1983 avec les valeurs calculées d'après les hypothèses de la fig. 14 .

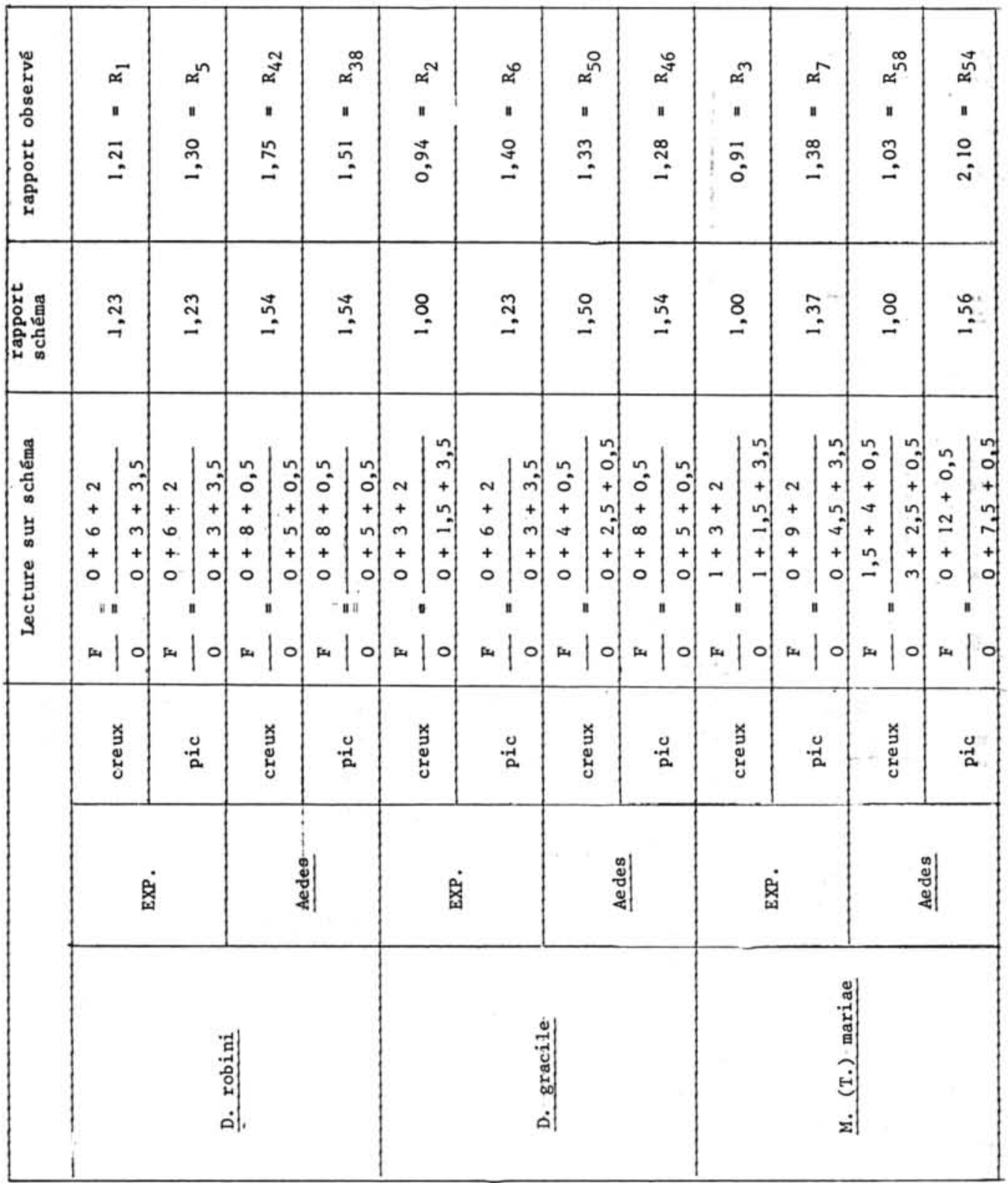

Le calcul théorique de la densité microfilarienne (colonne "lecture sur schéma ») est fait suivant l'exemple $D$. vobini - Expérimentateur - creux - flanc :

$0+6+2=[0$ croix $\times 1$ unité de vaisseau de moins de $15 \mu \mathrm{m}]$

$+[2$ croix $\times 3$ unités de vaisseaux de $15-70 \mu \mathrm{m}]$

$+[1$ croix $\times 2$ unités de vaisseaux de plus de $70 \mu \mathrm{m}]$ 


\section{E - Analyse des données concernant la queue}

La vascularisation de la queue d'un Cebidae comporte, nous l'avons vu (chap. III, C3), des particularités importantes : l'ensemble est riche en capillaires mais la partie plus antérieure comporte de gros vaisseaux à paroi musculaire épaisse et des glomi appliqués contre les vertèbres. Les vaisseaux de 15 à $70 \mu \mathrm{m}$ sont rares.

Il a été dit par ailleurs ce que ces particularités anatomiques entraînent : début du prélèvement, fin du prélèvement (chap. v B). La première goutte du prélèvement, dont le sang provient principalement de vaisseaux de moins de $15 \mu \mathrm{m}$, est extrêmement riche en microfilaires, particulièrement en Tetrapetalonema; la fin du prélèvement est un phénomène complexe puisque l'expérimentateur, pour obtenir la quantité de sang qui lui est nécessaire, doit comprimer les vaisseaux d'avant en arrière le long des vertèbres; il en résulte une congestion et un vidage mécanique des petits vaisseaux qui enrichit artificiellement le prélèvement en microfilaires de $M$. (T.) mariae.

Si ce massage est répété à intervalles de temps trop rapprochés, il semble y avoir " un phénomène de vidage ", le nombre des microfilaires nouvellement installées dans les fins vaisseaux ne compensant pas suffisamment l'extraction mécanique réalisée artificiellement. Les différentes données prennent un intérêt particulier dans le cas de $M$. (T.) colombiensis que nous examinons dans le sous-chapitre suivant.

Finalement les données qui peuvent foumir l'image la plus réelle sur la localisation vasculaire des microfilaires dans la queue sont fournies par les repas des Aedes. Les rapports d'ingestion $Q / O$ sont les suivants :

$$
\begin{array}{ll}
\text {-D. robini } & \text { - pic } \mathrm{R} 40=0,64 \\
\text { - } \text { - creux } \mathrm{R} 44=0,83 \\
\text { - gracile } & \text { - pic } \mathrm{R} 48=0,62 \\
- \text { creux } \mathrm{R} 52=0,63 \\
\text { - (T.) mariae: } \quad \text { - pic } \mathrm{R} 56=0,59 \\
\text { - creux R60 }=0,52
\end{array}
$$

Les prises de microfilaires à la queue par les Aedes sont constamment très inférieures à celles de l'oreille et donc a fortiori à celles du flanc.

Les résultats s'expliquent puisque le sang récolté par les Aedes dans la queue est, conformément au raisonnement suivi dans le chapitre précédent, pauvre en vaisseaux de 15-70 $\mu \mathrm{m}$ où se trouvent les microfilaires.

En opposition avec ces chiffres, les prises de sang faites par l'expérimentateur sont souvent très élevées et présentent, selon chaque espèce, de nombreuses anomalies apparentes.

\section{1 - D. robini (fig. 10, tableau XXV)}

Le sang prélevé par l'expérimentateur dans l'expérience du 30 septembre1 er octobre présente peu d'anomalies, sauf des nombres régulièrement plus élevés dans la queue que dans l'oreille. 

autres.

Le premier des rapports obtenus $(\mathrm{R} 17=1,06)$ est moins élevé que tous les

Dans les autres cas, en effet, le rapport avoisine le chiffre 2, conforme donc à celui obtenu après la pratique du massage.

Le nombre de $6 \mathrm{~h}$, le 30 septembre, n'est pas très différent de celui de $6 \mathrm{~h}$ le lendemain ; il n'y a pas le "phénomène de vidage ", que nous observerons pour les espèces qui vivent dans les plus petits vaisseaux.

\section{2 - D. gracile (fig. 11, tableau XXVI)}

Ce qui a été observé pour $D$. robini peut être à peu près répété pour $D$. gracile.

En effet, le rapport concernant le premier prélèvement est le plus faible; les autres prélèvements sont tous nettement plus élevés (effet du massage).

Les chiffres obtenus à $6 \mathrm{~h}$ le $1^{\mathrm{er}}$ octobre 1983 sont supérieurs à ceux de la veille à la même heure. Le pic de la microfilarémie, qui, chez cette espèce, est tardif, peut être retardé de 3 heures chez un animal tenu éveillé pendant toute la nuit (cf. chap. III).

\section{3 - M.(T.) mariae (fig. 12, tableau XXVII)}

Le " phénomène de massage " s'accentue pour cette espèce; le rapport, étant de 0,5 initialement, s'élève considérablement dans les prélèvements ultérieurs (il atteint 4 à $18 \mathrm{~h}$ ).

Le rapport entre les 2 prélèvements queue de $6 \mathrm{~h}$ du matin s'élève à 2,35 , ce qui objective bien également l'importance de ce phénomène de massage.

Il est même ici si important qu'il camoufle la faible périodicité de cettc: espèce, détectée à l'oreille.

\section{F - Cas particulier de M.(T.) colombiensis}

$M$.(T.) colombiensis n'a pu être analysée en même temps que les trois autres espèces puisque les densités de cette espèce dans les différentes expériences sont habituellement si faibles qu'aucun calcul ou rapport ne peut être considéré comme significatif.

L'analyse des particularités de cette espèce doit donc être effectuée par d'autres procédés.

Les données sûres qui concernent $M$.(T.) colombiensis sont les suivantes : a) Ses microfilaires sont abondantes sur les coupes histologiques dans les capillaires sous-épidermiques de l'oreille (en particulier sur la face ventrale) et de l'extrémité de la queue, c'est-à-dire dans des zones où le réseau capillaire est particulièrement dense.

Plus antérieurement sur la queue, et au flanc, lorsque le réseau capillaire devient plus lâche, ces microfilaires sont rares.

b) Elles sont assez abondantes dans des scarifications de l'oreille si celles-ci sont faites à la face ventrale. Ainsi, le 8 août 83 à. 21 h, juste après l'anesthésique, nous 
avons 179 microfilaires : 113 robini, 45 gracile, 17 mariae et 4 colombiensis. Le 2 août 1983, à 12 h, 3 heures après l'anesthésique, nous avons 22 microfilaires :

11 robini, 2 gracile, 3 mariae et 6 colombiensis.

c) Elı ’s ne sont prises qu'en quantité tout à fait minime par les Aedes quel que soit le lieu st l'heure du repas.

d) Le seul moment où l'espèce apparaisse en quantité importante dans le sang est l'expérience représentée dans le tableau $X X V I I I$ où il y a massage des organes examinés, oreille et surtout queue, qui sont comparés pendant un cycle nycthéméral.

Dans cette expérience le phénomène de massage se manifeste de façon prédominante : les rapports $Q / O$ (R20) atteignent couramment les valeurs de 5 et 7 , même une fois 40 (à $15 \mathrm{~h}$ ).

Un deuxième phénomène se superpose clairement au précédent.

A l'oreille comme à la queue, on constate régulièrement que les 2, 3 ou 4 premiers prélèvements sont supérieurs aux suivants et que ceux-ci décroissent ensuite plus ou moins régulièrement.

Après un certain nombre de prélèvements par massage, il y a donc, dans le cas de cette espèce, un phénomène de vidage.

Ayant vu par histologie combien la localisation de ces microfilaires est particulière, l'ensemble des phénomènes constatés pourrait s'expliquer assez simplement :

Les grosses microfilaires de $M$. (T.) colombiensis apparaissent en histologie immobilisées dans les capillaires sous-épidermiques. Le massage de l'organe parasité (oreille et queue) déloge ces animaux de leur microniche, du fait de la vaso-dilatation générale et de la pression manuelle exercée durant le massage. Les microfilaires vont donc se trouver repoussées dans les vaisseaux de plus gros calibre et reprises ensuite dans le courant rapide de la circulation générale avec les hématies et les microfilaires de petite taille.

On peut donc comprendre :

1) pourquoi ces microfilaires restent inaccessibles aux Aedes, alors que l'expérimentateur en trouve beaucoup au moment même du massage ;

2) pourquoi, après un certain nombre de masiages (environ 2 dans l'oreille, 3 ou 4 à la queue), la réserve se trouvant dans le-capillaires s'épuise, ne pouvant se renouveler au même rythme.

\section{G - Richesse en microfilaires des repas d'Aedes pris en phase de microfilarémie croissante ou décroissante}

La presque totalité de l'expérimentation a été faite à heures fixes pour que les résultats soient comparables entre eux, d'uue part le matin à $9 \mathrm{~h}$ solaire, d'autre par le soir à $21 \mathrm{~h}$ solaire (pic de la microfilarémı pour D. robini).

Il est devenu nécessaire de compléter ces données $ן$ 'ar quelques sondages effectués à des heures différentes. 


\section{A - Microfilarémie croissante}

L'expérimentation, tableau $X X X V I$, chap. vI, indique les résultats des prélèvements effectués successivement à $21 \mathrm{~h}, 12 \mathrm{~h}$ et $18 \mathrm{~h}$ après injection d'Imalgène aı début de chaque intervention.

On notera qu'à $21 \mathrm{~h}$ le nombre de microfilaires à l'oreille est anormalement bas (789 pour robini, 201 pour gracile, 177 pour mariae). Il est possible que le singe ait été dérangé au moment où il s'endormait et que son pic de microfilarémie ait été retardé.

Quoi qu'il en soit, on remarque que les rapports concernant les 2 espèces périodiques sont extrêmement élevés : il semble que lorsque la microfilarémie augmente trìs rapidement, le rapport soit particulièrement fort.

Pour $D$. robini le rapport R74 atteint 7 une demi-heure ou trois quarts d'heure après l'inoculation d'Imalgène à midi, donc au moment où la courbe va se redresser ; le rapport $\mathrm{R} 75$ monte à plus de 10 à $18 \mathrm{~h} 30$ au moment où l'effet de l'Imalgène injecté à $18 \mathrm{~h}$ et l'effet du pic naturel à $21 \mathrm{~h}$ s'additionnent.

Pour $D$. gracile, le rapport R76 est de 4,46 à $21 \mathrm{~h}$, donc au moment où la courbe de microfilarémie propre à cette espèce s'élève le plus vite. Elle reste à un taux plus faible $(1,70$ et 1,84$)$ à 12 h 30 et 18 h 30 ; dans ces 2 cas, la montée est plus tardive.

Pour M.(T.) mariae, à $21 \mathrm{~h}$ le rapport est celui attendu au pic $(\mathrm{R} 79=1,48)$; à $12 \mathrm{~h} 30$, la microfilarémie a beaucoup baissé et le rapport observé est celui du creux ; à 18 h 30 le rapport R81 s'élève à 1,90, peu avant la montée naturelle de la microfilarémie de cette espèce.

Les résultats de cette expérience paraissent très fortement positifs mais sont loin d'être rigoureux puisque pendant le temps qui sépare la ponction de sang du repas de l'Aedes, la microfilarémie s'élève fortement pour robini et gracile.

Une expérience plus rigoureusement contrôlée était donc nécessaire. Le sang a été prélevé une première fois aussitôt avant le repas des Aedes et une seconde fois aussitôt après le repas de ceux-ci. Le repas effectif a lieu en moyenne, non pas au moment même où les Aedes sont mis en contact avec le singe, mais plutôt à la fin du temps qui leur est alloué. Nous avons donc, pour permettre la comparaison avec le sang prélevé par les Aedes, pris arbitrairement le nombre de microfilaires comptées avant le repas plus les $2 / 3$ de la différence entre les densités microfilariennes avant et après le repas.

L'ensemble des rapports obtenus figure sur le tableau $X X X V I I$.

On constate que :

a) colombiensis a des chiffres trop faibles pour permettre une analyse ;

b) les trois autres espèces ont au début de l'expérience des rapports (R25, R26 et R27) qui correspondent à ceux que l'on peut attendre sachant que la microfilarémie au flanc est égale ou supérieure à celle de l'oreille et que, même au bout de 16 minutes après l'Imalgène, les taux de microfilarémie commencent à s'élever ;

c) dans les 3 cas à partir de ces nombres initiaux, les rapports s'élèvent. Pour robini, le sommet R86 est à 9 h 10. Pour gracile, le sommet est à 9 h 52 (R90). Dans les 
2 cas donc le rapport atteint son maximum au moment de la montée la plus rapide de la microfilarémie ;

d) dans le cas de mariae, l'évolution des rapports est comparable à celui de gracile alors que, dans ce cas, la microfilarémie chute de plus en plus rapidement. Le mécanisme paraît donc différent : ici, le rapport élevé de $M$.(T.) mariae peut s'expliquer par un retard des microfilaires, lors de la chute de la microfilarémie, à quitter les vaisseaux de 15-70 $\mu \mathrm{m}$, où elles sont freinées.

\section{$\mathrm{B}$ - Microfilarémie décroissante}

Les résultats de cette expérience (tableau XXXVIII) correspondent aux rapports $\mathrm{R} 97$ à $\mathrm{R} 100$.

Pour robini le rapport $\mathrm{R} 97=1,58$ correspond à $\mathrm{R} 38$ observé normalement au pic.

Pour gracile $\mathrm{R} 98=1,24$ correspond également à la valeur du pic $(\mathrm{R} 46=1,28)$.

Pour mariae R99 = 1,96 est élevé et semblable à celui de l'expérience précédente où la microfilarémie de mariae, au contraire de celles de robini et gracile, chutait. L'interprétation donnée ici est la même que précédemment.

De cet ensemble d'expériences, on peut conclure à une surcharge en microfilaires des repas d'Aedes effectués en phase de microfilarémie croissante dans le cas des 2 Filaires périodiques, et à une surcharge en phase de microfilarémie décroissante dans le cas de $M .(T$.$) mariae.$

Il s'agit dans tous les cas, semble-t-il, d'une accumulation passagère des microfilaires dans les vaisseaux d'environ 15-70 $\mu \mathrm{m}$ de diamètre.

\section{H - Rapport entre les prises de sang prélevé par l'Aedes et par l'expé- rimentateur}

Le rapport entre ce que prend le vecteur et ce que prend l'expérimentateur a un intérêt évident en épidémiologie d'autant plus que certains auteurs ont cru que les Hématozoaires pouvaient être attirés dans le sang prélevé par le vecteur par un supposé chimiotactisme positif pour la salive de celui-ci. Comme nous l'avons $\mathrm{vu}$, il s'agit malheureusement de la partie la plus délicate de l'expérimentation puisque le repas des vecteurs ne peut être instantané et que le taux de la microfilarémie peut varier de façon importante pendant un très court laps de temps.

Dans le sous-chapitre $\mathrm{D}$ nous avons donc utilisé les comparaisons ponction et ingestion Flanc/Oreille pour tester nos hypothèses ; ce test s'étant révélé favorable nous prendrons donc exactement les mêmes hypothèses et appliquerons les mêmes formules pour tenter de voir si les rapports entre les prises de sang prélevé par l'Aedes et par l'expérimentateur sont fiables et peuvent être admis en dépit des difficultés expérimentales (tableau XLII).

1 - Examen des rapports R21-22-23 et R29-30-31, concernant la prise de l'Aedes piquant au flanc par rapport à celle de l'expérimentateur faite au même endroit.

L'ensemble des données est regroupé sur le tableau XLII en haut. 
Nous obtenons :

- 0,94 au lieu de 1,06 pour robini - creux (R21);1, 24 au lieu de 1,06 pour robini — pic (R29).

- 1,11 au lieu de 0,9 pour gracile - creux (R22); 1,07 au lieu de 1,06 pour gracile - pic (R30) ;

- 0,95 au lieu de 1,00 pour mariae - creux (R23); et 0,88 au lieu de 1,13 pour mariae — pic (R31).

Les divergences observées sont inférieures à $20 \%$ et nous paraissent acceptables. Nous conclurons donc qu'au flanc les prises de sang faites par le vecteur ne diffèrent pas considérablement de celles qui sont faites par l'expérimentateur.

2 - Examen des rapports R25-26-27 et R33-34-35, concernant la prise de l'Aedes piquant au flanc par rapport à celle de l'expérimentateur faite à l'oreille.

L'ensemble des données est regroupé sur le tableau XLII en bas.

Nous obtenons :

- 1,38 au lieu de 1,30 pour robini - creux (R25); 1,62 au lieu de 1,30 pour robini - pic (R33) ;

- 1,45 au lieu de 0,9 pour gracile - creux (R26); 1,32 au lieu de 1,30 pour gracile — pic (R34) ;

- 1,35 au lieu de 1,00 pour mariae - creux (R27) ; 1,46 au lieu de 1,56 pour mariae - pic (R35).

Deux nombres sont clairement en discordance; ce sont ceux qui concernent le "creux garcile " et le "creux mariae". Si l'on se rapporte aux tableaux $X V I$ et $X V I I$ (chap. v) il apparaît que, dans les 2 cas, il s'agit de données exceptionnelles puisque 4 fois le rapport des microfilarémies $\mathrm{F} / \mathrm{O}$ est inférieur à 1 et une fois seulement (le cas de cette expérience) il est positif.

On ne peut donc attacher beaucoup de valeur à ces données. Il est utile de savoir le caractère approximatif des mesures faites en prenant le sang à l'oreille lorsque l'on tente d'apprécier le nombre de microfilaires prélevé par les Aedes qui se nourrissent sur toute la surface du corps.

Nous avons vu qu'une appréciation par le sang pris à la queue apporterait des incertitudes encore plus grandes.

\section{Chapitre VIII : Reprise des problèmes posés par les Filaires du Saïmiri à l'aide de modèles fournis par d'autres hématozoaires}

Nous avons essayé d'établir dans les pages précédentes : a) que le taux de parasitisme est différent dans le sang provenant de très fins vaisseaux, de petits vaisseaux ou de gros vaisseaux; b) que la composition d'un prélèvement sanguin varie dans la proportion relative des hématies provenant de ces 3 catégories de vaisseaux selon qu'il est prélevé au flanc, à l'oreille ou à la queue et selon qu'il est prélevé par le moustique ou par l'expérimentateur. 
TABleau XLII. - En haut, rapports Aedes / Expérimentateur (EXP.) des prélèvements faits au flanc; en bas, rapports entre les prélèvements faits au flanc par l'Aedes et ceux faits à l'oreille par l'expérimentateur : comparaison des valeurs obtenues avec les valeurs calculées d'après les hypothèses de la fig. 14 .

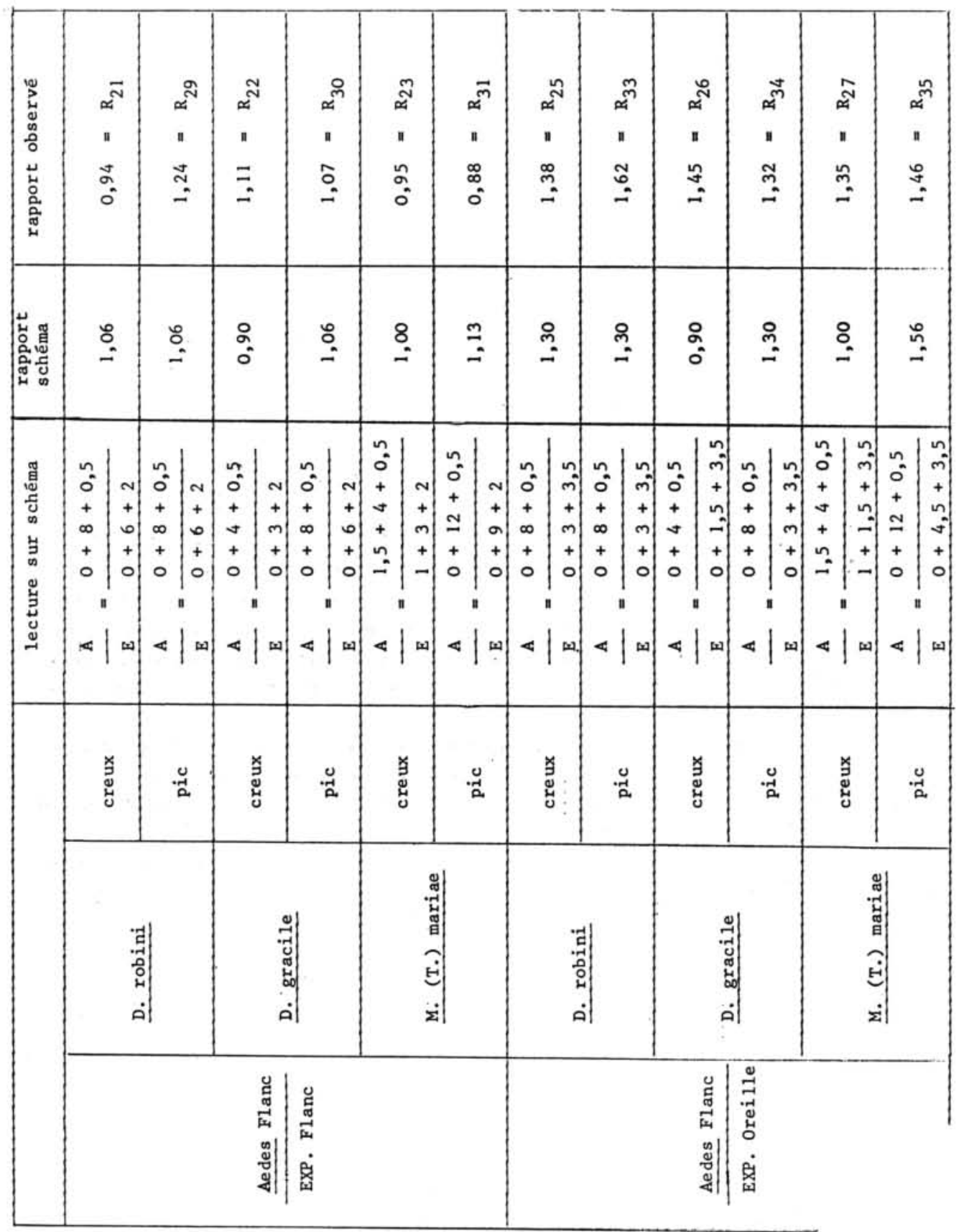


Ces particularités aboutissent à de nombreuses " anomalies " dans les résultats expérimentaux telles que le phénomène de la surcharge en microfilaires en phase de microfilarémie croissante, le " phénomène de la première goutte ", la localisation élective de certains hématozoaires dans les petits vaisseaux.

Nous voudrions dans ce présent chapitre :

a) tenter de confirmer et de préciser certains des résultats précédents grâce à l'emploi d'autres modèles biologiques,

b) tenter de résoudre certains problèmes déjà posés par d'autres hématozoaires à l'aide des notions mises en évidence dans ce travail.

\section{A - Phénomène de la surcharge en microfilaires pendant la phase de microfila- rémie croissante : Filaires de Corvidés}

Dans le chapitre précédent, la richesse en microfilaires des repas d'Aedes pris en phase de microfilarémie crcissante ou décroissante est d'appréciation difficile du fait que les paramètres étudiés sont particulièrement instables. Les données foumies par un couple hôte-parasite totalement différent, nous paraissent donc utiles à présenter.

Un Corvus frugilegus, capturé en région parisienne en janvier 1981, est parasité par des microfilaires d'Eufilaria sp. Les données morphologiques et le cycle évolutif réalisé expérimentalement chez Culicoides nubeculosus seront publiés prochainement (Bain, Petit et Grolleau).

Le rythme nycthéméral est intéressant car le pic est à $6 \mathrm{~h}$ solaire le 18 février, il est à $3 \mathrm{~h}$ solaire le 17 mars et, le 4 mai, il se dédouble en un premier pic situé à $21 \mathrm{~h}$ et un $2^{\mathrm{e}}$ pic à $4 \mathrm{~h}$ solaire ( $\mathrm{fig} .15$ ).

Des Aedes ont été gorgés dans la nuit du 4 au 5 mai 1981 aux heures suivantes:

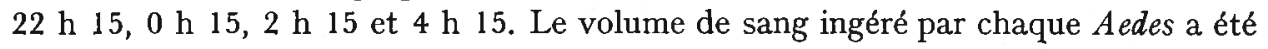
estimé à $4,5 \mathrm{~mm}^{3}$ (conformément aux prises effectuées à cette époque dans d'autres expériences). Les résultats sont consignés sur le tableau XLIII et sur la figure 15.

Tableau XLIII. - Ingestion par A. aegypti des microfilaires d'Eufilaria sp., en phase de microfilarémie croissante ou décroissante. 4-5 mai 1981.

\begin{tabular}{lcccc}
\hline & 22 h 15 & 0 h 15 & 2 h 15 & 4 h 15 \\
\hline Mf I T / A & $180+/-9$ & $291+/-13$ & $214+/-15$ & $130+/-11$ \\
Mf I T / 10 mm & $399+/-9$ & $647+/-13$ & $475+/-15$ & $288+/-11$ \\
Mf VB / $10 \mathrm{~mm}^{3}$ & $488+/-10$ & $319+/-10$ & $360+/-10$ & $498+/$ - 10 \\
R & 0,82 & 2,03 & 1,32 & 0,58 \\
\hline
\end{tabular}

Mf I T $=$ nombre de microfilaires ingérées sur le thorax

Mf $\mathrm{V} \mathrm{B}=$ nombre de microfilaires à la veine brachiale

$\mathrm{A} \quad=$ Aedes 


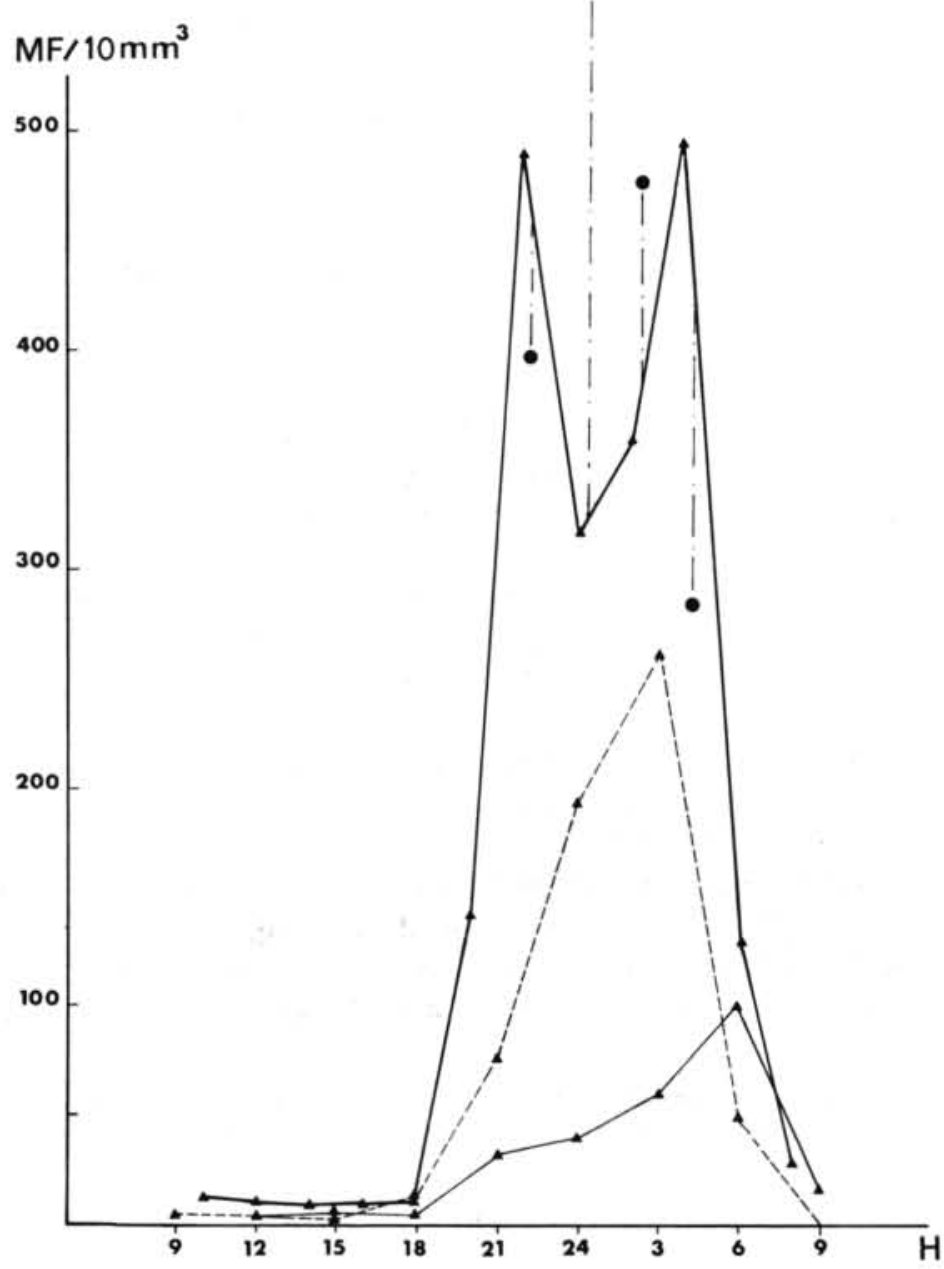

FIG. 15. - Corvus frugilegus - Eufilaria sp. :-- Variations nycthémérales de la microfilarémie à 3 dates différentes, les I 8 et I9 février I98I (trait plein fin), les I 7 et I 8 mars I98I (trait pointillé), les 4 et 5 mai 1981 (trait plein épais). - Nombre de microfilaires ingérées par $A$. aegypti, en phase de microfilarémie croissante ou décroissante. Chaque point noir sur la figure représente la valeur du nombre moyen de microfilaires ingérées (rapporté à Io $\mathrm{mm}^{3}$ de sang), dans quatre gorgements effectués respectivement à $22 \mathrm{~h}$ I $5,6 \mathrm{~h} \mathrm{I5}, 2 \mathrm{~h} \mathrm{I} 5$ et $4 \mathrm{~h} \mathrm{I} 5$ dans la nuit du 4 au 5 mai I98I. 
Quatre comptages ont donc été effectués :

Le $I^{\text {er }}$ à 22 h 15 correspond au moment où la microfilarémie commence sa décroissance après le premier pic ; $R$, rapport entre nombre de microfilaires ingérés par l'Aedes et nombre de microfilaires comptées dans le sang par l'expérimentateur, est égal à 0,82 .

Le $2^{\mathrm{e}}$ comptage, à $0 \mathrm{~h} 15$, coïncide avec le moment où la microfilarémie recommence à croître pour arriver au deuxième pic nocturne ; $\mathrm{R}$ est très élevé : 2,03 .

Le 3 e comptage, à 2 h 15 , correspond à la partie moyenne de la courbe ascendante de la microfilarémie; $\mathrm{R}$, égal à 1,32, est moins élevé que précédemment mais reste très largement positif.

Le $4^{\mathrm{e}}$ comptage enfin, effectué à $4 \mathrm{~h} 15$, correspond au moment où la microfilarémie chute brutalement après le $2^{\mathrm{e}}$ pic nocturne; le rapport est alors très faible : 0,58 .

Ces résultats sont plus faciles à lire que ceux du Saïmiri du fait qu'il y a 2 pics nocturnes. Ils confirment bien la notion d'une surcharge en microfilaires des vaisseaux de 15 à $70 \mu \mathrm{m}$ de diamètre, c'est-à-dire ceux qui précèdent la dichotomie vasculaire au-delà de laquelle les microfilaires ne peuvent plus s'engager. L'Aedes, qui pique électivement dans ces vaisseaux, a donc une surcharge notable en microfilaires au moment où celles-ci sont libérées massivement par les poumons (début de la phase de microfilarémie croissante).

Un point reste à préciser. Le phénomène de surcharge en microfilaires pendant la phase de microfilarémie croissante n'a pas été étudié dans le cas de faibles microfilarémies; on pourrait imaginer que l'encombrement des vaisseaux étant moindre, le phénomène soit moins marqué ou disparaisse.

Par ailleurs la Filaire du corbeau montre beaucoup plus clairement que ne l'ont fait les Filaires du Saïmiri la sous-ingestion par l'Aedes pendant la phase de microfilarémie décroissante.

\section{B - « Phénomène de la première goutte de sang »; comparaison des petits et des grands repas dans les cas des Plasmodiums de Rongeurs}

A l'extrémité de la queue du Saïmiri, tissu particulièrement riche en fins vaisseaux, les rapports $\mathrm{R} 9$ à $\mathrm{R} 12$, montrent que la première goutte de sang prélevée par l'expérimentateur, si elle est de faible volume, est beaucoup plus riche en microfilaires que ne l'est le sang qui est recueilli ultérieurement. Cela est particulièrement net pour $M$.(T.) mariae qui fréquente les fins vaisseaux, mais sans y être immobilisé comme $M$.(T.) colombiensis. Il était donc intéressant de vérifier le phénomène avec un autre modèle pour lequel nous soupçonnons également une concentration dans les vaisseaux de 10 à $20 \mu \mathrm{m}$ de diamètre.

Nous avons constaté qu'au cours de leur repas les moustiques font très fréquemment plusieurs essais dans de fins vajsseaux avant de trouver le plus gros vaisseau ou la petite flaque d'hémorragie qui permet la complète réplétion. Il était donc intéressant et facile de comparer les infestations de moustiques ayant fait des petits 
repas avec ceux qui ont fait de gros repas. Car ceux qui ont fait de petits repas ont nécessairement en moyenne une proportion plus importante de sang de "1 re goutte " que n'en ont en moyenne ceux qui ont fait de gros repas.

En prenant pour modèle le paludisme de rongeur, nous avons donc comparé le rendement des Anophèles bien gorgés et peu gorgés.

Deux expériences ont été réalisées de la même façon. De nombreux Anopheles stephensi sont nourris sur une souris blanche infectée depuis 3 jours par Plasmodium yoelii yoelii, souche $17 \mathrm{x}$. On sait que cette souche est particulièrement infestante à ce jour mais que les gamétocytes infectants (type I de Landau et coll. 1979) qui sont grands $(10,5 \mu \mathrm{m}$ de diamètre) sont paradoxalement très rares à cette date dans le sang prélevé par l'expérimentateur (Landau et coll., 1979).

Les Anophèles sont divisés en trois lots : lot non gorgé ; lot très peu gorgé ; lot très bien gorgé.

Tableau XLIV. - Plasmodium yoelii yoelii infection des Anopheles en fonction du poids du repas de sang.

\begin{tabular}{|c|c|c|c|c|c|}
\hline & & $\begin{array}{l}\text { Nb A } \\
\text { pesés }\end{array}$ & $\begin{array}{l}\text { Poids } \\
\text { mg/A }\end{array}$ & $\begin{array}{l}\mathrm{Nb} A \\
\text { disséqués }\end{array}$ & $\begin{array}{l}\text { Nb d'oocystes } \\
\text { par A }\end{array}$ \\
\hline \multirow[t]{2}{*}{ EXP. I } & $\begin{array}{l}\text { Non gorgés } \\
\text { Petits repas } \\
\text { Gros repas }\end{array}$ & $\begin{array}{l}50 \\
59 \\
51\end{array}$ & $\begin{array}{l}2,6 \\
2,2 \\
3,9\end{array}$ & $\begin{array}{l}\overline{49} \\
36\end{array}$ & $\begin{array}{c}-\overline{30 \pm 22} \\
46 \pm 17\end{array}$ \\
\hline & \multicolumn{5}{|c|}{ Rendement petits repas / gros repas $=6,52$} \\
\hline \multirow[t]{2}{*}{ EXP. II } & $\begin{array}{l}\text { Non gorgés } \\
\text { Petits repas } \\
\text { Gros repas }\end{array}$ & $\begin{array}{l}50 \\
51 \\
58\end{array}$ & $\begin{array}{l}1,8 \\
1,96 \\
3,45\end{array}$ & $\begin{array}{l}\overline{40} \\
39\end{array}$ & $\begin{array}{l}-\overline{26 \pm 9} \\
91 \pm 7\end{array}$ \\
\hline & \multicolumn{5}{|c|}{ Rendement petits repas / gros repas $=2,85$} \\
\hline
\end{tabular}

$\mathrm{A}=$ Aedes $-\mathrm{Nb}=$ nombre

Les expériences sont assez imprécises, car les moustiques n'ont pas été conservés à jeun et ont eu l'opportunité dans les cages d'élevage de prélever ou non un peu de jus sucré. Ainsi dans l'expérience 1 les Anophèles non gorgés pèsent un peu plus lourd que les Anophèles peu gorgés.

Dans l'expérience 2, le gros repas $(3,45 \mathrm{mg}-1,8 \mathrm{mg}=1,65 \mathrm{mg})$ est 10 fois plus lourd que le petit repas (1,96 $\mathrm{mg}-1,80 \mathrm{mg}=0,16 \mathrm{mg}$ ).

Nous admettons donc que le repas moyen des Anophèles peu gorgés correspond environ au $1 / 10^{\mathrm{e}}$ du repas des très bien gorgés.

Le rapport entre rendement des petits repas et rendement des gros repas est donc de $: 30 \times 10 / 46=6,52$ dans la première expérience et $26 \times 10 / 91=2,85$ dans la deuxième. 
Ce résultat positif confirme bien l'hypothèse selon laquelle certains hématozoaires dont la taille est de l'ordre de $10 \mu \mathrm{m}$ se concentrent dans les très fins vaisseaux (10 à $20 \mu \mathrm{m}$ de diamètre).

Ces fins vaisseaux n'interviennent pratiquement pas lorsque le sang est prélevé par l'expérimentateur; ils interviennent relativement peu, dans les gros repas qui correspondent le plus souvent aux cas où l'Anophèle trouve un vaisseau plus large.

\section{C — Problème des hématozoaires dans les petits vaisseaux de moins de $15 \mu \mathrm{m}$}

Les résultats d'expériences variées nous ont fait admettre que $M$.(T.) mariae, contrairement à $D$. gracile et à $D$. robini, séjourne dans les fins vaisseaux, au moins au cours des périodes de basse microfilarémie. Elle n'y est pas partiellement emprisonnée, comme nous l'avons vu pour $M$.(T.) colombiensis, mais paraît au contraire pouvoir en être extraite aisément soit par ponction expérimentale (" phénomène de la première goutte de sang ", à l'extrémité de la queue) soit encore mieux par succion au moment du début de la piqûre du moustique.

Un problème du même ordre s'est posé précédemment dans l'étude du paludisme des Rongeurs.

Landau et coll. (1979) ont montré que les gamétocytes de Plasmodium yoelii subissent une évolution en 4 types morphologiques 0 , I, II, III. Ce sont les gamétocytes $\mathrm{I}$, les plus grands (9 à 10,5 $\mu \mathrm{m}$ de diamètre), qui sont infectants. Comme il a été dit en $\mathrm{B}$ le fait paradoxal vient de ce que l'Anophèle les prélève essentiellement le $3^{\mathrm{e}}$ jour de l'infection chez la Scuris blanche, alors qu'à ce moment on n'en voit presque pas dans le sang circulant.

Pour chercher à résoudre ce problème, nous avons (1981) injecté à des Rats blancs des microsphères inertes de 8 à $24 \mu \mathrm{m}$ de diamètre et avons étudié la façon dont elles étaient ingérées par des moustiques, $24 \mathrm{~h}$ après l'inoculation.

Nous espérions démontrer une ingestion privilégiée des grosses particules, ce qui était suggéré far les travaux de plusieurs auteurs (Pichon et coll., 1975, 1980, Petit, 1978) pour rendre compte des surdispersions observées. Dans les hypothèses formulées à l'époque, cela aurait pu être attribué à un freinage des gros parasites dans les fins vaisseaux.

Nous avions considéré l'expérience comme négative puisque l'Aedes ou l'Anophèle n'ingèrent pas préférentiellement les particules les plus grosses (Petit, 1981). Nous pensons aujourd'hui qu'il ne faut pas centrer notre intérêt sur les particules de $20 \mu \mathrm{m}$ et plus mais au contraire sur celles de $12 \mu \mathrm{m}$ de diamètre. Les données obtenues à cette époque sont donc reprises sur le tableau XLV et sur les histogrammes figure 16*.

Une différence significative permet d'opposer d'une part la taille des particules dans le sang du cœur ou du sinus oculaire, et d'autre part la taille des particules du sang prélevé par l'Aedes ou l'Anophèle.

* Dans la publication de I98I, les calculs et les moyennes sont exacts mais l'histogramme "sinus de l'œil " comporte une erreur de dessin pour les classes Io et I2 $\mu \mathrm{m}$ (se reporter au tableau $X L V)$. 

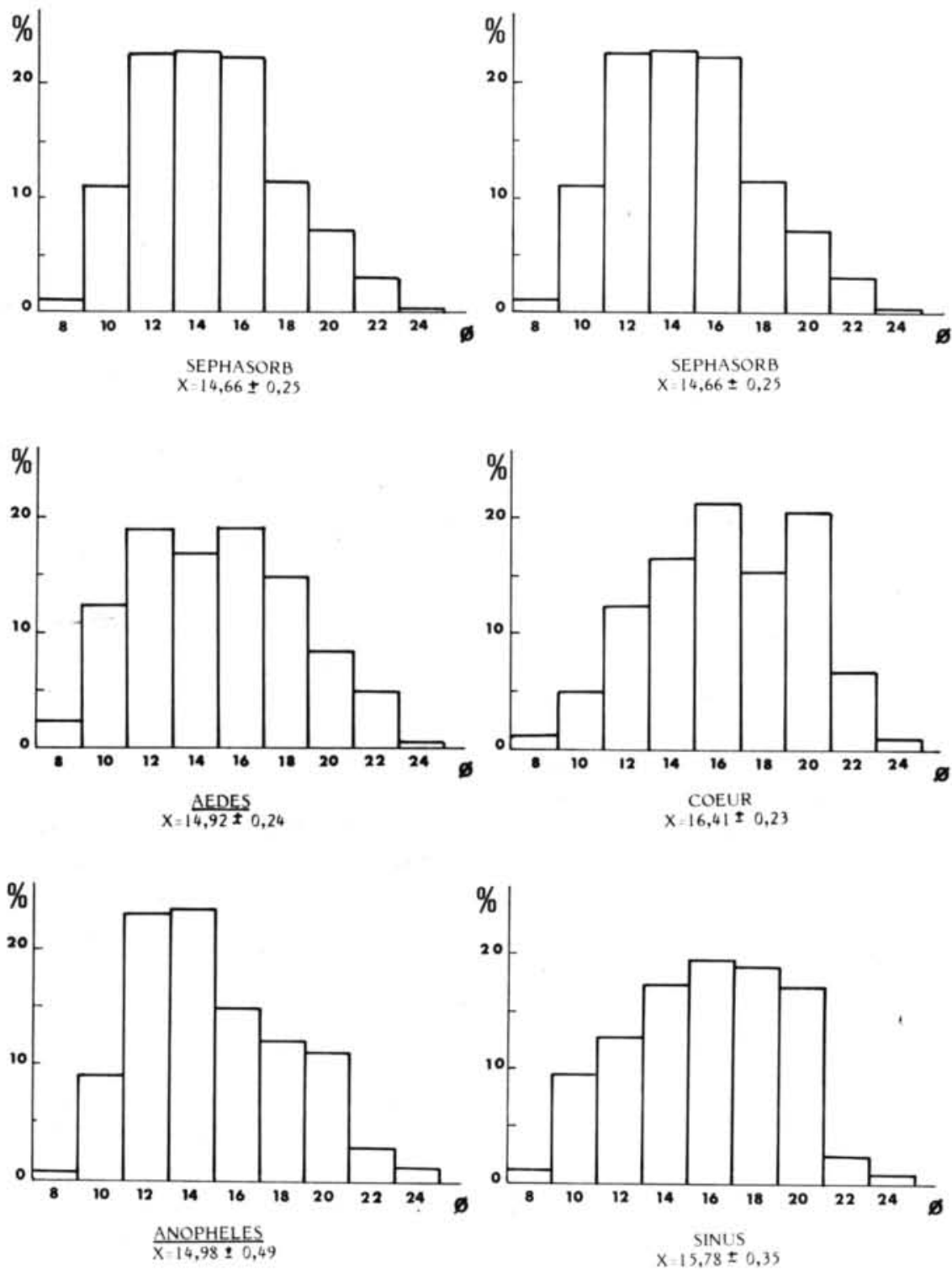

Fig. I6. - Histogramme de fréquence des microsphères en fonction de leur diamètre (fréquence exprimée en pourcentage) : dans le produit pur (Sephasorb) : dans le sang d'une souris blanche (cœur, sinus oculaire) après injection du Sephasorb; dans le sang ingéré par les Aedes (IO4 우) et les Anopheles ( 30 \%) gorgés sur cette souris. ( $\mathrm{X}=$ diamètre moyen des microsphères, avec intervalle de confiance à $5 \%$ ). 
C'est précisément sur les particules de $12 \mu \mathrm{m}$ de diamètre que porte la différence.

En regroupant l'ensemble des particules de 8 à $14 \mu \mathrm{m}$ nous avons les pourcentages suivants :

- cœur $34,98 \%+/-3,33 \%$ * et sinus oculaire $40,65 \%+/-5,15 \%$, donc plus faible que le produit initial $(56,20 \%+/-3,79 \%)$.

- Anophèle 56,70\%+/-7,49\% et Aedes 51,12\%+/-3,84\% donc peu différents du produit initial.

Il résulte du travail effectué sur les microfilaires du Saïmiri que la portion du système circulatoire dont le diamètre est inférieur à $15 \mu \mathrm{m}$ n'est pas prélevé par l'expérimentateur.

Le sang contenu dans cette partie du système circulatoire n'apparaît que lorsque le moustique, n'atteignant pas du premier coup un gros vaisseau, exerce une véritable succion sur les petits vaisseaux; il absorbe ainsi électivement les parasites accumulés dans ces microbiotopes.

Les particules inertes de $12 \mu \mathrm{m}$ de diamètre ou les gamétocytes infectants de $10 \mu \mathrm{m}$ de diamètre s'accumulent donc dans ces très fins vaisseaux et ne sont prélevés qu'électivement par la succion du moustique lors du repas.

La prise préférentielle des particules inertes de $12 \mu \mathrm{m}$, ou celle de gamétocytes infectants dont le diamètre est proche, est en accord avec cette notion.

TABLEAU XLV. - Fréquence des microsphères en fonction de leur diamètre (en pourcentage) :

- dans le produit pur (Sephasorb).

- dans le sang d'une souris blanche (cœur, sinus oculaire) après injection du Sephasorb.

- dans le sang ingéré par les Aedes (104 O) et les Anopheles (30 O) gorgés sur cette souris.

\begin{tabular}{rrrrrr}
\hline$\varnothing$ & Sephasorb & Aedes & Anopheles & Coeur & Sinus \\
\hline 8 & 0,91 & 3,20 & 0,60 & 1,01 & 1,15 \\
10 & 10,62 & 12,48 & 9.15 & 5,06 & 9,70 \\
12 & 22,26 & 18,80 & 23,17 & 12,39 & 12,32 \\
14 & 22,41 & 16,64 & 23,78 & 16,43 & 17,48 \\
16 & 21,95 & 19,26 & 15,24 & 21,49 & 19,48 \\
18 & 11,59 & 15,25 & 12,19 & 15,42 & 19,19 \\
20 & 6,86 & 8,47 & 11,59 & 20,48 & 17,19 \\
22 & 3,20 & 5,24 & 3,05 & 6,32 & 2,29 \\
24 & 0,15 & 0,61 & 1,22 & 1,39 & 1,15 \\
\hline
\end{tabular}

* Intervalle de confiance à $5 \%$, Schwartz p. 47 . 


\section{Conclusions}

L'expérimentation faite sur un Saïmiri parasité par 4 espèces de Filaires nous amène aux conclusions suivantes :

a) que la densité des parasites dans le système circulatoire varie selon le diamètre des vaisseaux;

b) que le sang prélevé par le vecteur ou par l'expérimentateur est un mélange provenant de vaisseaux de petit, moyen et grand diamètres dont la proportion relative varie suivant l'organe où il est prélevé et suivant le mode de prélèvement.

Nous distinguons trois sortes de Filaires :

1 - Espèces à périodicité marquée et ne pénétrant pas dans les vaisseaux de moins de $15 \mu \mathrm{m}$.

La grande espèce $D$. robini et la petite espèce $D$. gracile sont les 2 représentants de cette catégorie.

Les microfilaires s'accumulent dans les poumons pendant le jour. Lorsqu'elles sont lâchées dans la circulation en très grand nombre, elles suivent passivement les hématies dans les grands vaisseaux; cependant lorsque l'arbre circulatoire effectue ses dernières dichotomies, les microfilaires ne peuvent suivre les hématies et doivent s'acheminer par un réseau d'anastomoses où elles se trouvent transitoirement concentrées, cela tout au moins lorsqu'il y a une forte microfilarémie.

On explique donc aisément pourquoi les prélèvements au flanc (riche en vaisseaux de 15 à $70 \mu \mathrm{m}$ ), et les repas d'Aedes (qui se gorgent de préférence sur ce type de vaisseaux) sont plus riches que les autres et cela surtout au moment de la phase croissante de la microfilarémie.

La seule différence appréciable entre $D$. gracile et $D$. robini est que $D$. gracile, plus petite, s'écoule plus facilement et ne présente ce phénomène d'accumulation dans les moyens vaisseaux qu'au moment du pic.

2 - Espèce périodique avec microfilaires pouvant pénétrer transitoirement dans les vaisseaux de moins de $15 \mu \mathrm{m}$.

L'exemple en est fourni par $M$.(T.) mariae. Les preuves de sa présence dans les très fins vaisseaux sont données par la distribution moins poissonnienne de cette espèce et surtout par le " phénomène de la première goutte de sang ": le premier $\mathrm{mm}^{3}$ qui s'écoule de l'extrémité de la queue est particulièrement riche en sang provenant des très fins vaisseaux et extrêmement riche aussi en $M .(T$.) mariae.

Les différents rapports obtenus expérimentalement paraissent indiquer que cette fréquentation des très fins vaisseaux ne s'effectue que pendant le creux de la microfilarémie ; pendant le pic au contraire l'espèce paraît, encore plus que les précédentes, s'accumuler dans le réseau de 15-70 $\mu \mathrm{m}$ de diamètre.

On peut imaginer que le corps de cette microfilaire, très long et facilement pelotonné sur lui-même, résiste aisément au courant sanguin dans les vaisseaux de moins de $70 \mu \mathrm{m}$ de diamètre. 
3 - Espèce non périodique avec microfilaires vivant à l'intérieur des capillaires.

$M .(T$.$) colombiensis vit dans les très fins vaisseaux et le nombre de microfilaires$ circulantes est infime par rapport à la population globale. Le seul procédé pour l'obtenir en quantité importante consiste à masser l'oreille et surtout la queue de façon à déloger les microfilaires des capillaires. Le sang recueilli à cet instant est donc riche en microfilaires, mais de façon artificielle et transitoire car les microfilaires sont vite reprises dans la circulation générale et l'on peut observer un " phénomène de vidage "à l'oreille et à la queue lorsque plusieurs massages sont effectués successivement.

Nous interprétons donc cette espèce comme une Filaire à microfilarémie sanguine encore mal adaptée à cette biologie, puisqu'elle n'est prélevée que très difficilement par le vecteur, que ce soit un moustique, un culicoïde ou une tique.

Ces conclusions sont fondées essentiellement sur la comparaison entre la richesse du sang prélevé au flanc par rapport à celui prélevé à l'oreille pour chacune des 3 espèces de Filaires à microfilaires circulantes, pendant le creux ou pendant le pic de la microfilarémie, que le prélèvement soit effectué par l'expérimentateur ou par l'Aedes ( $f$ g. 1 et 14).

Les douze rapports obtenus ont été considérés comme fiables puisqu'il s'agit de rapports entre deux mesures qui peuvent être faites simultanément.

C'est donc essentiellement pour satisfaire à la valeur numérique de ces douze rapports que les hypothèses précédentes ont dû être adoptées.

Les autres données expérimentales sont moins fiables car, pour des raisons matérielles, les 2 termes du rapport ne peuvent être obtenus simultanément ; cependant aucune d'entre elles ne contredit les données précédentes et les hypothèses formulées.

Il est regrettable que l'ensemble ne puisse être confirmé par l'observation directe; cela est impossible puisqu'un stress quelconque, et a fortiori la mort de l'animal, entraîne des modifications vasculaires inévitables.

Il reste pourtant quelques procédés pour tester la valeur des hypothèses formulées.

I - Entreprendre des expériences du même type avec un matériel biologique différent.

Cela a été fait avec une Filaire très particulière parasite de Corvus frugilegus; le résultat est positif.

II - Expérimenter sur les conséquences que cette hypothèse implique.

Cela a été réalisé en comparant la richesse relative en gamétocytes de Plasmodium de Rongeurs des petits repas et des grands repas du vecteur, Anopheles stephensi; le résultat est positif.

III - Tenter de comprendre à l'aide des hypothèses précédentes certains phénomènes biologiques déjà constatés mais non encore expliqués.

Nous avons pris le phénomène paradoxal de Landau et coll., montrant qu'une infectivité maximale pour l'Anophèle du sang du Rongeur paludéen (3e jour de 
l'infection dans nos conditions expérimentales) correspond à une période où l'expérimentateur ne voit pas encore les gamétocytes circuler dans le sang.

L'expérience réalisée avec des microsphères inertes inoculées au Rongeur montre l'enrichissement relatif du repas du vecteur en particules de $12 \mu \mathrm{m}$ de diamètre.

Nous avons constaté directement par ailleurs la dépression des vaisseaux rythmée par le pompage lors du repas du vecteur. Tout concorde donc pour admettre que les parasites d'environ $10 \mu \mathrm{m}$ de diamètre (taille des gamétocytes infectants) puissent s'accumuler dans les très fins vaisseaux et n'en être délogés que par l'aspiration faite par le vecteur lors de son repas.

Il résulte de ces considérations que l'ingestion des microfilaires par l'Aedes (et peut-être de façon plus générale l'ingestion d'un hématozoaire par son vecteur) n'est pas sous la dépendance d'adaptation particulière telle qu'un chimiotactisme. C'est la richesse en parasites du sang ingéré qui se retrouve passivement chez le vecteur mais cette richesse est très variable puisqu'elle dépend des facteurs complexes que nous venons d'analyser.

\section{BIBLIOGRAPHIE}

Anderson R. M. : The regulation of host population growth by parasitic species. Parasitology, I978, 76, II9-I 57

AshbURN P. M., CRAIG C. F. : Observation upon Filaria philippinensis and its development in the mosquito. Philipp. J. Sci., 1907, B, 2, I-5.

Barn O. : Remarques au sujet d'une nouvelle Filaire de Caméléon malgache, proche de Foleyella brevicauda. Bull. Mus. Natn. Hist. Nat., $2^{\mathrm{e}}$ sér., I968, 40, 802-806.

Bain O., Baker M., Chabaud A. G. : Nouvelles données sur la lignée Dipetalonema (Filarioidea, Nematoda). Ann. Parasitol. Hum. Comp., 1982, 57, 593-620.

Bain O., Petit G., Grolleau G. : Développement chez Culicoides nubeculosus d'Eufilaria spp., Filaires parasites de Corvidés. Ann. Parasitol. Hum. Comp. (sous presse).

Bain O., Petit G., Rosales-Loesener L. : Les Filaires parasites de Singes sud-américains. Bull. Mus. Natn. Hist. Nat., (sous presse).

Bertram D. S. : Studies on the transmission of cotton rat Filariasis. I. The variability of the intensities of infection in the individuals of the vector, Liponyssus bacoti, its causation and its bearing on the problem of quantitative transmission. Ann. Trop. Med. Parasitol., I949, 43, $3 \mathrm{I} 3-332$.

Bliss C. I., Fisher R. A. : Fitting the negative binomial distribution to biological data. Biometrics, I953, 9. I 76-200.

Boorman J. P. T. : Observations on the Feeding habits of the mosquito Aedes (Stegomyia) aegypti (L); The loss of fluid after a blood meal and the amount of blood taken during Feeding. Ann. Trop. Med. Parasitol., I960, 54, 8-14.

Briegel H., LeA A. O., Klowden M. J. : Hemoglobinometry as a method for measuring blood meal sizes of mosquitoes (Diptera : Culicidae). J. Med. Entomol., I979, I 5, 235-238.

BuRTON G. J. : The intake of microfilariae of Wuchereria bancrofti by Culex pipiens fatigans in British Guiana. Ann. Trop. Med. Parasitol., I964, 58, 333-338.

Carme B. : Étude des variations de la microfilarémie dans la Filariose à Loa loa. Ann. Soc. Belge Med. Trop., 1983, 63, 333-339.

Crans W. J. : Experimental infection of Anopheles gambiae and Culex pipiens fatigans with Wuchereria bancrofti in coastal East Africa. J. Med. Entomol., I973, Io, I89-193.

Dollfus R.-Ph., Chabaud A. G. : Cinq espèces de Nématodes chez un Sapajou (Ateles ater) (G. Cuvier) mort à la ménagerie du Muséum. Arch. Mus. Nat., I955, 3, 27-40.

Duke B. O. L. : The uptake of the microfilariae of Acanthocheilonema streptocerca by Culicoides grahamii and their subsequent development. Ann. Trop. Med. Parasitol., I954, 48, 416-420.

DuкE B. O. L. : The intake of the microfilariae of Acanthocheilonema perstans by Culicoides grahamii and C. inornatipennis, and their subsequent development. Ann. Trop Med. Parasitol. 1956, so, 32-38. 
Dunn F. L., Lambrecht F. L. : On some Filarial parasites of South American Primates, with a description of Tetrapetalonema tamarinae $\mathrm{n}$. sp., from the Peruvian Tamarin Marmoset, Tamarinus nigricollis (Spix, 1823). J. Helmintol., I963, 37, 261-286.

EBerha RD M. L. : Tetrapetalonema (T.) mystaxi sp. n. (Nematoda : Filarioidea) from Brazilian Moustached Marmosets, Saguinus M. mystax. J. Parasitol., 1978, 64, 204-207.

Eberhard M. L., Lowrie R. C., Oriher T. C. : Development of Dipetalonema gracile and $D$. caudispina to the infective stage in Culicoides hollensis. J. Parasitol., 1979, 6), 89-95.

Esslinger J. H. : Dipetalonema obtusa (McCoy, I936) comb. n. (Filarioidea : Onchocercidae) in Colombian primates, with a description of the adult. J. Parasitol., I966, 52, 498-502.

Esslinger H. H. : Tetrapetalonema (T.) panamensis (McCoy, I936) comb. n. (Filarioidea : Onchocercidae) in Colombian primates, with a description of the adults. J. Parasitol., I979, $65,924-927$

Esslinger J. H. : Tetrapetalonema (T.) saimiri sp. n. (Nematoda : Filarioidea) from Colombian squirrel monkeys, Saimivi sciureus. J. Parasitol., I98I, 67, 268-27I.

Esslinger J. H. : Tetrapetalonema (T.) colombiensis sp. n. (Nematoda : Filarioidea) from Colombian primates. $J$. Parasitol., I982, 68, I I 38 -I I4I.

FAust E. C. : Notes on Helminths from Panama. III. Filarial Infection in the Marmosets, Leontocebus geoffroyi (Pucheran) and Saimiri orstedii (Reinhardt) in Panama. Trans. Roy. Soc. Trop. Med. Hyg., $1935,28,627-634$.

Freitas J. F. T. : Achegas Helminthologicas. R. Ci. Biol. Belem, I964, 2, 3-4o.

GORDON R. M., LumSDEN W. H. R. : A study of the behaviour of the mouth-parts of mosquitoes when taking up blood from living tissue ; together with some observations on the ingestion of microfilariae. Ann. Trop. Med. Parasitol., 1939, 33, 259-278.

GrifFITHS R. B., Gordon R. M. : An apparatus which enable the process of feeding by mosquitoes to be observed in the tissues of a live rodent ; together with an account of the ejection of saliva and its significance in Malaria. Ann. Trop. Med. Parasitol., I952, 46, 3I I-3I9.

Gubler D. J., Inui T. S., Black H. R., Bhattacharya N. C. : Comparisons of microfilaria density in blood sampled by finger-prick, venipuncture and ingestion by mosquitoes. $A m$. J. Trop. Med. Hyg., I973, 22, I $74-178$

Hawning F. : Dirofilaria magnilarvatum Price, I959 (Nematoda : Filarioidea) from Macaca irus Cuvier. III. The behaviour of the microfilariae in the mammalian host. J. Parasitol., I959, 4), 5 I I-5I 2 .

HAwKING F. : The responses to various stimuli of microfilariae of Dirofilaria corynodes, of Dipetalonema marmosetae and of unidentified species of Filariae in Saimiri sciureus and Cacajao monkeys. Int. J. Parasitol., I973, 3, 433-439.

Hopkins C. A., Nicholas W. L. : Culicoides austeni, the vector of Acanthocheilonema perstans. Ann. Trop. Med. Parasitol., 1952, 46, 276-283.

Jordan P., Goatly K. D. : Bancroftian filariasis in Tanganyika : a quantitative study of the uptake, fate and development of microfilariae of Wucherevia bancrofti in Culex fatigans Ann. Trop. Med. Parasitol., 1962, 56, I 73-187.

Kershaw W. E., BeEsley W. N., Crewe W. : Studies on the intake of microfilariae by their insect vectors, their survival, and their effect on the survival of their vectors. VI : Further observations on the intake of the microfilaria of Loa loa and Acanthocheilonema perstans by Chrysops silacea in laboratory conditions : the pattern of the intake of a group of Flies. Ann. Trop. Med. Parasitol., I955, 49, II4-I20.

Kershaw W. E., CRewe W., BeEsley W. W. : Studies on the intake of microfilariae by their insect vectors, their survival, and their effect on the survival of their vectors. II : The intake of the microfilariae of Loa loa and Acanthocheilonema perstans by Chrysops spp. Ann. Trop. Med. Parasitol., 1954, 48, I02-I09.

Kershaw W. E., Lavoipierre M. M. J., Beesley W. N. : Studies on the intake of microfilariae by their insect vectors, their survival, and their effect on the survival of their vectors. VII : Further observations on the intake of the microfilariae of Dirofilaria immitis by Aedes aegypti in laboratory conditions: The pattern of the intake of a group of flies. Ann. Trop. Med. Pavasitol., I955, 49, 203-2 I I.

Kershaw W. E., Lavoipierre M. M. J., Chalmers T. A. : Studies on the intake of microfilariae by their insect vectors, their survival, and their effect on the survival of their vectors. I. Dirofilaria immitis and Aedes aegypti. Ann. Trop. Med. Parasitol., I953, 47, 207-224.

Knotт J. : A method for microfilarial surveys on day blood. Trans. Roy. Soc. Trop. Med. Hyg., I939, 33, I9I-I97.

Lagraulet J., Barsinas M., Fagneaux G. : Hétérogénéité de la répartition des microfilaires dans le sang périphérique chez les malades atteints de Filariose de Bancroft et aperçu général sur la Filariose aux Marquises. Bull. Soc. Pathol. Exot., 1972, 65, 698-703.

Landau I., Miltgen F., Boulard Y., Chabaud A. G., Baccam : Études sur les gamétocytes des Plasmodiums du groupe "vivax ": morphologie, évolution, prise par les Anophèles et infectivité des microgamétocytes de Plasmodium yoelii. Ann. Parasitol. Hum. Comp., I979, 54, I $45-\mathrm{I} 6 \mathrm{I}$. 
LAVOIPIERRE M. M. J. : Studies on the host- parasite relationships of Filarial nematodes and their arthropod hosts. II. The arthropod as a host ot the nematode : A brief appraisal of our present knowledge based on a study of the more important literature from I878 to I957. Ann. Trop. Med. Parasitol., 1958, 52, 326-345.

Mac Coy : Filarial parasites of the Monkeys of Panama. Am. J. Trop. Med., I936, I6, 383-403.

Mac Fie J. W. S., Corson J. F. : A new species of Filarial larva found in the skin of Natives in the Gold Coast. Ann. Trop. Med. Parasitol. r922, I6, 383-403.

Mac Greevy P. B., Kolstrup N., Tao J., Mac Greevy M. M., Marshall T. P. de C. : Ingestion and development of Wucherevia bancrofti in Culex quinquefasciatus, Anopheles gambiae and $A$ edes aegypti after feeding on humans with varying densities of microfilariae in Tanzania. Trans. Roy. Soc. Med. Hyg., I982, 76, 288-295.

Mac Mahon J. E., Marshall T. F., Vauchan J. P., Abaru D. E. : Bancroftian Filariasis : a comparison of microfilariae counting techniques using counting chamber, standard slide and membrane (nucleopore) filtration). Ann. Trop. Med. Parasitol., I979, 73, 457-464.

Manson-BaHR P. H. : Filariasis and elephantiasis in Fiji. Res. Mem. Lond. Sch. Trop. Med., I $912,1,1-8$.

Nathan M. B., Raccurt C. : Higher concentrations of microfilariae in capillary blood from the ear lobe than from the finger in Wucherevia bancrofti and Mansonella ozzardi infections. Trans. Roy. Soc. Trop. Med. Hyg., I979, 73, 455-457.

Nicholas W. L., Gordon R. M., Kershaw W. E. : The taking up of microfilariae in the blood by Culicoides spp. Trans. Roy. Soc. Trop. Med. Hyg., 1952, 46, 377.

Nicholas W. L., Kershaw W. E. : Studies on the intake of microfilariae by their insect vectors, their survival, and effect on the survival of their vectors. III. : The intake of the microfilariae of Acanthocheilonema perstans by Culicoides austeni and C. grahamii. Ann. Trop. Med. Pavasitol., I954, 48, 201-206.

O'Connor F. W., BeATTy H. A. : The abstraction by Culex fatigans of Microfilaria bancrofti from man. J. Trop. Med. Hyg., I937, 40, IOI-IO3.

Pacheco G., Atkins M. J., GuRIan J. : Quantification of infection of Ticks with Dipetalonema vitae. J. Parasitol., I972, $58,275^{-278}$.

Peel E., Chardome M. : Sur des Filaridés de Chimpanzés "Pan paniscus " et "Pan satyrus "au Congo belge. Ann. Soc. Belge Med. Trop., I946, 26, i I 7-1 56.

Petit G. : La Filaire Dipetalonema dessetae : phénomènes de régulation et rendement parasitaire chez 1'Aedes vecteur. Ann. Parasitol. Hum. Comp., 1978, 53, 649-668.

Petit G. : Ingestion des microfilaires par le vecteur dans le cas d'une Filaire périodique Dipetalonema dessetae. Ann. Parasitol. Hum. Comp., I981, 56, 531-539.

Petit G., Bain O., Rousilhon C. : Deux nouvelles Filaires chez un singe Saimiri sciureus au Guyana. Ann. Parasitol. Hum. Comp, 1985, 60, 65-81.

Petit G., Pichon G. : Ingestion des microfilaires par le vecteur dans le cas des basses miciofilarémies. Ann. Parasitol. Hum. Comp., 1982, $57,73-78$.

Pichon G., Prod'hon J., Riviere F. : Distribution des microfilaires ingérées par les moustiques.

WHO. FIL., I 975,75, I $39,24 \mathrm{p}$.
PichoN G., ProD'hoN J., Riviere F. : Hétérogénéité de l'ingestion des parasites sanguicoles par leurs vecteurs : description quantitative et interprétation. C.R. Acad. Sci. Paris, I98o, 290 , sér. D., IOII-1013.

PRICE D. L. : Dirofilaria magnilarvalum n. sp. (Nematoda : Filarioidea) from Macaca irus Cuvier. I. Description of the adult Filarial worms. J. Parasitol., I959, 45, 499-504.

RamachandRan C. P. : Biological aspects in the transmission of Brugia malayi by Aedes aegypti in the laboratory. $J$. Med. Ent., I966, 3, 239-252.

Ramachandran C. P., ZaINI M. A. : Studies on the transmission of subperiodic Brugia malayi by Aedes (Finlaya) togoi in the laboratory. I. The intake and migration of microfilariae. Med. J. Malaya, 1967, 22, 1 36-144.

Rosen L. : Observations on the epidemiology of human Filariasis in French Oceania. Am. J. Hyg., I955, 6I, 2 I $9-248$.

ROYCHOWDHURY S. P., KANAIN A. M., DAS M. : Intake of microfilariae of Wucherevia bancrotti and Dirofilaria repens by Culex pipiens fatigans and Aedes aegypti respectively. J. Com. Dis., I969, $1,107-114$.

Samarawickrema W. A., Fola-Sone, Kazuyo-Ichimori, Cummings R. F. : Filariasis in Western Samoa. I. Transmission studies of Aedes (Stegomya) polynesiensis (Marks) and Aedes (Finlaya) samoanus (Gruenberg) on carriers with different levels of microfilaraemia of Wucherevia bancrofti. VHO.VBC.7975I, I979, 2 I p.

Schmid W. D., Robinson E. J.: The pattern of a host-parasite distribution. J. Parasitol., I972, S8, 907-9I0.

Schwartz D. : Méthodes statistiques à l'usage des médecins et des biologistes. Flammarion Médecine Sciences, Paris, I977, $318 \mathrm{p}$.

Southwood T. R. E. : Ecological methods. Chapman and Hall, London, I97 , 524 p.

Tanaka H., Chiba T., Tanaka H. : Quantitative observations on the ingestion of the microfilariae of Litosomoides carinii by its intermediate host Ornithonyssus bacoti. Jap. J. Parasit., I963, I2, I9I-I 95 . 
Webber W. A. F. : Dirofilaria aethiops Webber, 1955, a Filarial parasite of monkeys. III : The larval development in mosquitoes. Parasitology, 1955, 45, 388-400.

WhaRTON R. H. : Studies on filariasis in Malaya : observations on the development of Wuchereria malayi in Mansonia (Mansonioides) longipalpis. Ann. Trop. Med. Parasitol., I957, 51, 278295.

Wharton R. H. : Dirofilaria magnilarvatum Price, I959 (Nematoda : Filarioidea) from Macaca irus Cuvier. IV. Notes on larval development in Mansonioides mosquitoes. J. Parasitol., I959, 45, 5 $13-5$ I 8 .

WhARTON R. H. : Studies on Filariasis in Malaya : Field and laboratory investigation of the vectors of a rural strain of Wucherevia bancrofti. Ann. Trop. Med. Parasitol., I960, 54, 78-9I.

Yorke W., Blacklock B. : Observations on the Periodicity of Microfilaria nocturna. Ann. Trop. Med.Parasitol., 1917, II, I27-148.

Z. ELKE E. : Experimentelle untersuchungen uber die quantitativen Vernhältnisse bei der Übertragung von Dirofilaria immitis durch Stechmücken. Z. Tropenmed. Parasitol., 1973, 24 , 2I4-22I. 